\title{
Argonne Below Ground Model Part II: Population Dynamics, Exposure, and Fomite Transport
}

Decision and Information Sciences Division 


\begin{abstract}
About Argonne National Laboratory
Argonne is a U.S. Department of Energy laboratory managed by UChicago Argonne, LLC under contract DE-AC02-06CH11357. The Laboratory's main facility is outside Chicago, at 9700 South Cass Avenue, Argonne, Illinois 60439. For information about Argonne and its pioneering science and technology programs, see www.anl.gov.
\end{abstract}

\title{
DOCUMENT AVAILABILITY
}

Online Access: U.S. Department of Energy (DOE) reports produced after 1991 and a growing number of pre-1991 documents are available free via DOE's SciTech Connect (http://www.osti.gov/scitech/)

Reports not in digital format may be purchased by the public from the National Technical Information Service (NTIS):

U.S. Department of Commerce

National Technical Information Service

5301 Shawnee Rd

Alexandra, VA 22312

www.ntis.gov

Phone: (800) 553-NTIS (6847) or (703) 605-6000

Fax: (703) 605-6900

Email: orders@ntis.gov

Reports not in digital format are available to DOE and DOE contractors from the Office of Scientific and Technical Information (OSTI):

U.S. Department of Energy

Office of Scientific and Technical Information

P.O. Box 62

Oak Ridge, TN 37831-0062

www.osti.gov

Phone: (865) 576-8401

Fax: (865) 576-5728

Email: reports@osti.gov

\section{Disclaimer}

This report was prepared as an account of work sponsored by an agency of the United States Government. Neither the United States Government nor any agency thereof, nor UChicago Argonne, LLC, nor any of their employees or officers, makes any warranty, express or implied, or assumes any legal liability or responsibility for the accuracy, completeness, or usefulness of any information, apparatus, product, or process disclosed, or represents that its use would not infringe privately owned rights. Reference herein to any specific commercial product, process, or service by trade name, trademark, manufacturer, or otherwise, does not necessarily constitute or imply its endorsement, recommendation, or favoring by the United States Government or any agency thereof. The views and opinions of document authors expressed herein do not necessarily state or reflect those of the United States Government or any agency thereof, Argonne National Laboratory, or UChicago Argonne, LLC. 


\section{Argonne Below Ground Model Part II: Population Dynamics, Exposure, and Fomite Transport}

by

J.C. Liljegren and D.F. Brown

Decision and Information Sciences Division

Argonne National Laboratory

August 2014 



\section{Contents}

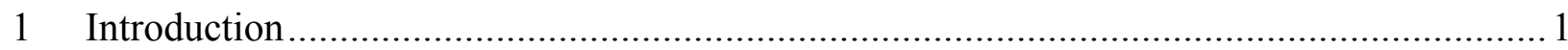

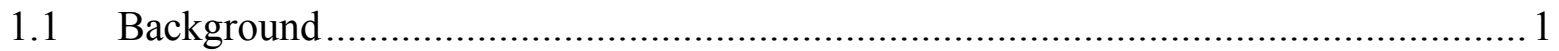

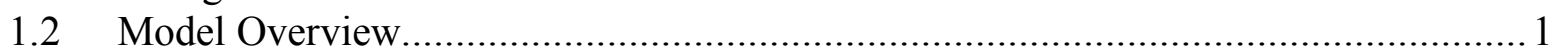

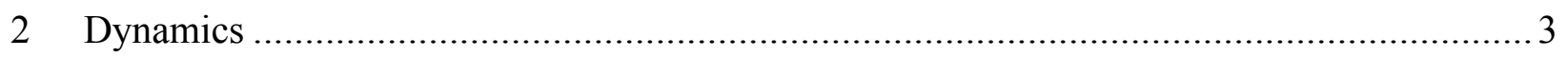

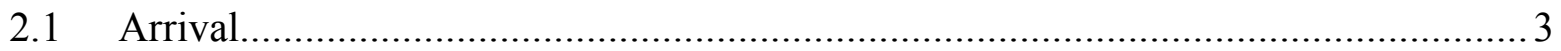

2.2 Destination Selection and Routing ................................................................. 5

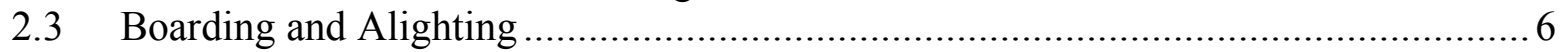

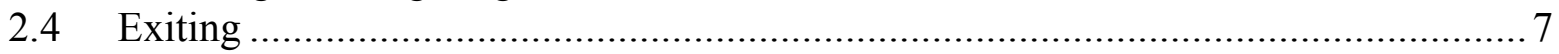

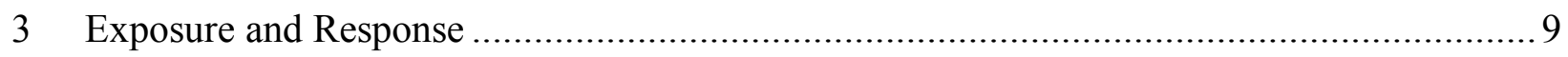

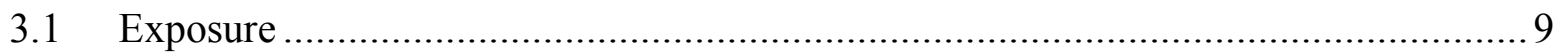

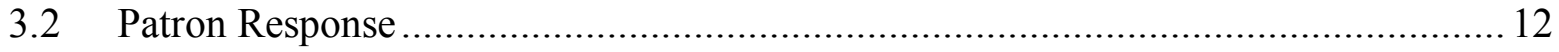

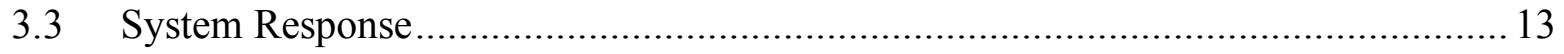

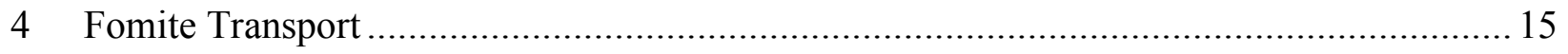

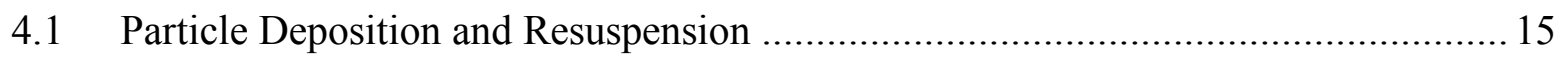

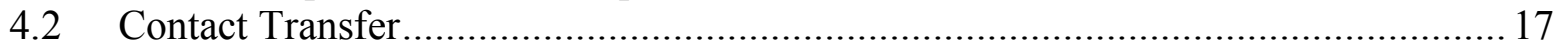

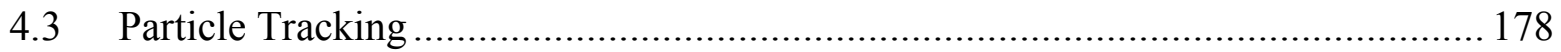

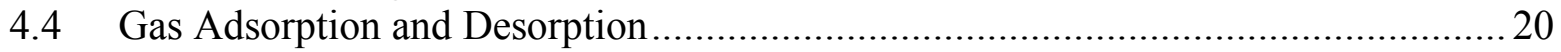

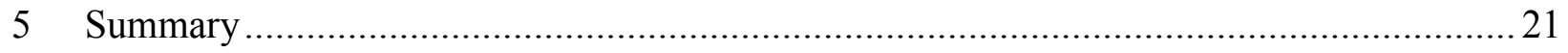

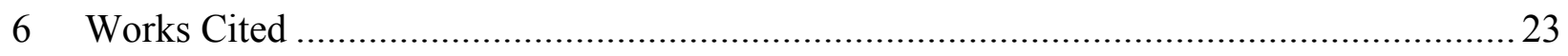

Appendix A: Analysis of NYCTA and PATH Turnstile Data................................................2

Appendix B: Calculating Origin-Destination Pairing ……...................................................29

Appendix C: Probit Analysis and Dose-Response Models.......................................................... 33

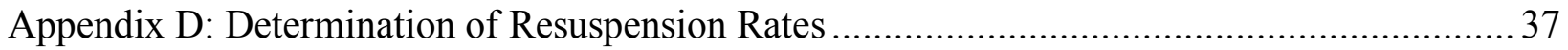

\section{Figures}

1 Hourly entry and exit trends for a central station and a terminus station............................. 4

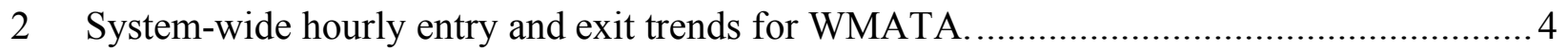

3 Actual and derived hourly entries for three WMATA stations........................................ 5

4 A typical side-platform station showing the platforms, platform exits, mezzanines, and

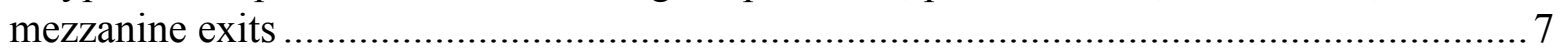

5 The dose-response curve for lethal exposure to anthrax if untreated.................................. 10

6 Number of unexposed, less-than-fatally exposed, and fatally exposed patrons following a bioagent release at $08: 30$ in a nearby station 


\section{Figures (Cont.)}

7 AEGL exposures and fatal exposures at a station in the central business district following the simulated release of sarin to illustrate the mitigating effects of

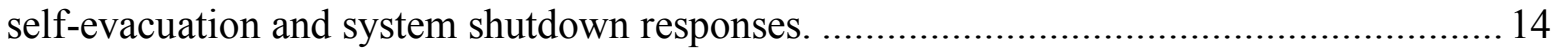

8 Dosage along a subway line for a gas and for different size particles without and

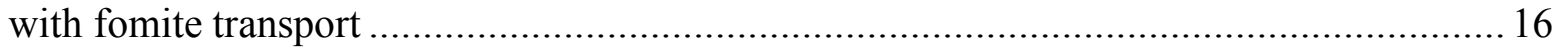

9 Number of colony-forming units deposited per unit area on the southbound platforms and tracks without and with particle tracking ............................................ 20

10 Passenger entries and exits for the Flatbush Avenue Station in Brooklyn .......................26

11 Passenger entries and exits for the Fulton Street Station in lower Manhattan...................26

12 Average hourly weekday entries and exits for the New York City subway......................27

\section{Tables}

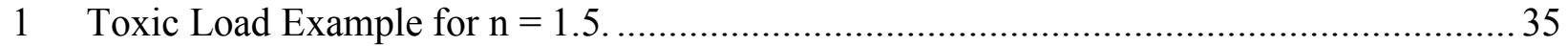

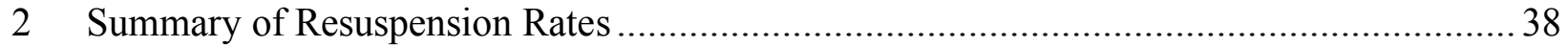




\section{Introduction}

\subsection{Background}

The Argonne Below Ground Model (BGM) was developed to provide a comprehensive simulation capability for analysis of subway threats at the level of the whole system. As described in Part I (Brown and Liljegren 2014), BGM predicts the movement and spread of a chemical or biological agent within an underground subway system as well as the amount of material released to the environment through station entrances, street-level vents, and tunnel portals. To assess the health consequences of an attack, BGM incorporates an agent-based population-effects component, described in this report, in which the movement of each person in the system is independently modeled, allowing their individual exposure, their resulting health effects, and their behavior (in the case of chemical exposure) to be determined.

\subsection{Model Overview}

Subway passengers may be exposed to chemical or biological agents during three phases of their trip: (1) as they arrive and wait for a train on the station platform, (2) as they ride the train, and (3) as they exit the station at their destination. Phases 1 and 2 may be repeated if passengers must transfer from one train to another to reach their destination. In a chemical attack, passengers may react in response to the characteristics of the agent and their level of exposure, and their collective reaction may subsequently influence system operation, e.g., station(s) may be evacuated and train operations may be altered or halted. Although passengers would be unaware of and therefore unable to react to a biological attack, bio-particles may deposit on passengers and then be shed (re-aerosolized or resuspended) throughout their trip and potentially even after they exit the subway. In effect, passengers may become mobile sources of bioagent ("fomite transport"). In addition, bio-particles deposited on station or train car floors may be resuspended by the action of passengers' footsteps, or they may adhere to the passengers' shoes and be redeposited elsewhere. The passenger model accounts for all of these possibilities.

The modeling approach is to follow individual passengers through the subway system to assign each passenger an entry station with an arrival time and a destination station derived from actual turnstile data, and then to determine their exposure (and possible reaction) as they wait for a train, ride the train, transfer to another train, if necessary, and finally exit the system. Passengers are also assigned individualized dose-response parameters to reflect the range of responses in an actual population; also, individualized particle deposition and tracking parameters are assigned to reflect the range of extant clothing and shoe types. 
This page intentionally left blank. 


\section{Dynamics}

\subsection{Arrival}

Subway patrons are assumed to arrive at each station randomly, following a Poisson process wherein the probability of $N$ persons arriving in a given hour is (Papoulis 1984, p. 210)

$$
P(N)=\left(e^{-\lambda} \lambda^{N}\right) / N !
$$

and $\lambda$ is the average number of arrivals for this hour. The time increment $\Delta t$ between successive arrivals in this hour is also random with a probability (Papoulis 1984, p. 362)

$$
P(\Delta t)=\lambda e^{-\lambda \Delta t}
$$

At the beginning of each hour in the simulation, $t_{0}$, random time increments obeying the above probability density are generated based on the average number of arrivals during that hour for each station: $\Delta t=-1 / \lambda \ln (1-r)$, where $\mathrm{r}$ is a uniform random number $[0,1)$. The arrival time of the $n^{\text {th }}$ patron for a given station is calculated as

$$
t_{n}=t_{o}+\sum_{i=1}^{n} \Delta t_{i} \text {, until } t_{\mathrm{n}}-t_{\mathrm{o}}>1 \text { hour. }
$$

For many subway systems, the average number of arrivals $\lambda(t)$ and exits each hour is determined for each station from average hourly statistics derived from turnstile data. (The analysis of the turnstile data for the New York City Transit Authority (NYCTA) and Port Authority TransHudson (PATH) subways is presented in Appendix A.) The exit statistics are adjusted as needed to ensure that the total number of arrivals and exits in a day are equal, i.e., the subway is neither a source nor a sink for people.

For subways systems for which only daily entry statistics are available, $\lambda(t)$ is estimated for each station by combining the hourly trends for a typical central station in the downtown business district, and a typical commuter station at a line terminus is weighted according to the location of the station between the central and terminus stations: $\lambda(t)=N f(t)$, where $N$ is the (known) daily total patron entries at a station and $f(t)$ is the interpolated hourly trend (hourly fraction of daily entries):

$$
f(t)=a(\xi) f_{C}(t)+[1-a(\xi)] f_{T}(t)
$$

where $\xi=\left(x-x_{C}\right) /\left(x_{T}-x_{C}\right)$ is the relative location of the station at $x$ between the central station at $x_{\mathrm{C}}(\xi=0)$ and the terminus station at $x_{T}(\xi=1)$. The interpolating function is $a(\xi)=$ $\sin (\xi \pi / 2)$. The entry and exit trends for the central and terminus stations are derived from turnstile data for Metro Center and Largo Town Center stations, respectively, in the Washington Metropolitan Area Transit Authority (WMATA) subway, and are presented in Figure 1. For Largo, the terminus station, arrivals peak during the morning travel period, whereas for Metro Center, the central station, entries peak during the evening travel period. Hourly exits exhibit the opposite trends with Largo exits peaking during the evening travel period and Metro Center exits peaking during the morning period. 
The results are normalized to ensure that the total number of daily entries for the system is correct, and that the hourly fraction of the total entries and exits match the observed trends for the WMATA system. The WMATA trends, presented in Figure 2, exhibit a typical pattern of peak entries and exits during the morning and evening travel periods, with entries leading exits.
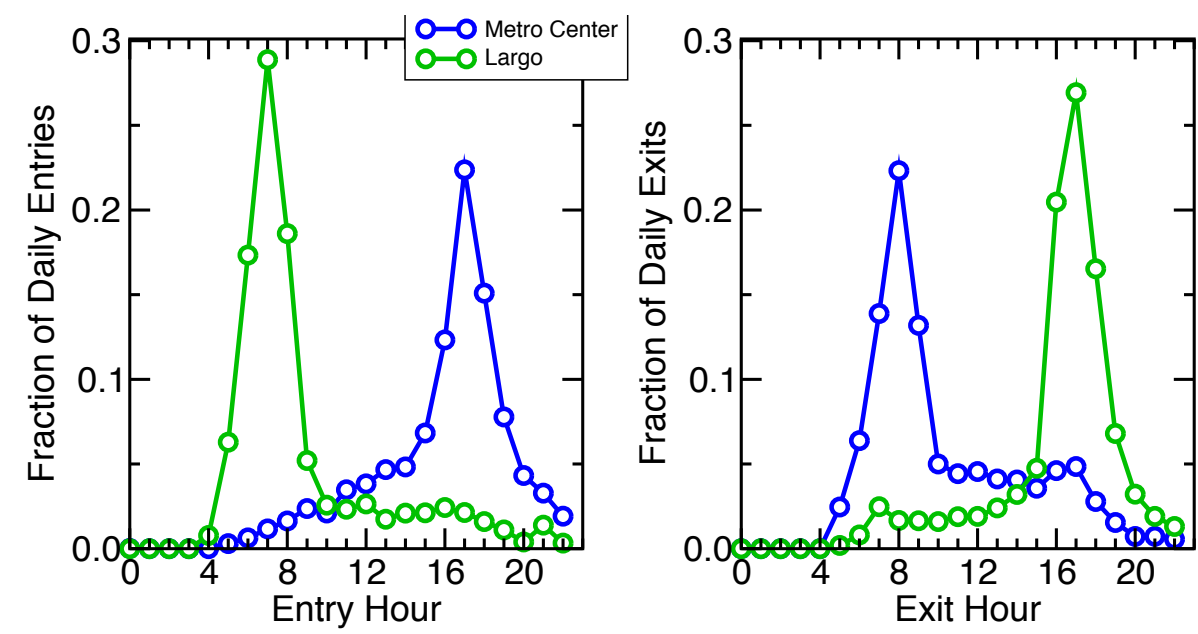

Figure 1. Hourly entry (left) and exit (right) trends for a central station (Metro Center) and a terminus station (Largo).

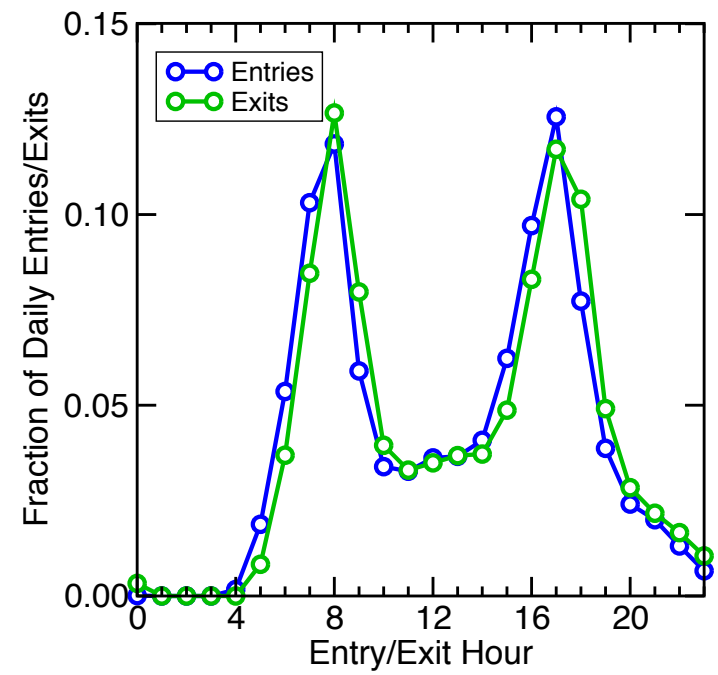

Figure 2. System-wide hourly entry and exit trends for WMATA.

The actual and derived trends for three intermediate stations $(\xi=0.11,0.33$, and 0.52$)$ in the WMATA system are compared in Figure 3, which reveals the reasonably good fidelity of the algorithm. When this algorithm is used to estimate hourly exits, the number daily exits at each station is assumed to equal the number of daily entries. This implicitly assumes all patrons make round trips, ultimately returning to their initial station of entry. 

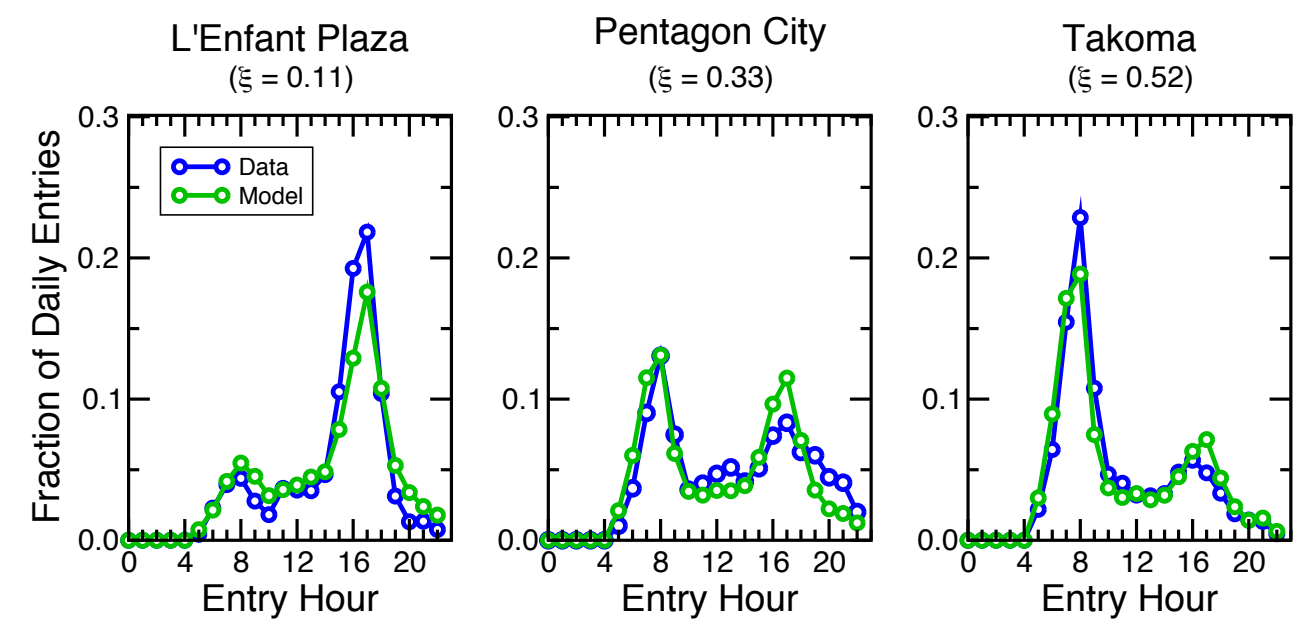

Figure 3. Actual and derived hourly entries for three WMATA stations.

\subsection{Destination Selection and Routing}

To assign a destination to each person, the likelihood that people arriving at a specified station during a given hour subsequently exit the subway at another specified station must be determined first, for each possible pair of arrival and destination stations. This is expressed as the probability of exiting at the $i^{\text {th }}$ station after having entered at the $j^{\text {th }}$ station, assuming that people do not exit at the same station they entered $(i \neq j)$

$$
P(i \mid j)=\frac{N_{i}}{N-N_{j}} ; i \neq j
$$

where $N=\sum N_{i}$ is the total number of people exiting the subway during this hour. (If the number of transfers between different subway lines is available, the algorithms in Appendix B may be used to determine $P(i \mid j)$.) The distribution function for this hour is

$$
F(i \mid j)=\sum_{k=1}^{i} P(k \mid j) .
$$

A destination is assigned to each passenger arriving at the $j^{\text {th }}$ station during this hour by generating a uniform random number $0 \leq \mathrm{r} \leq 1$ and finding the station $i$ that satisfies $r \leq F(i \mid j)$ for $i \neq j$.

Once each passenger is assigned a point of origin and a destination, their initial routing is assigned - the line they should take and whether they need to transfer to another line. The initial routing between station pairs is calculated separately from the simulation based on the fewest (preferably zero) transfers and then the fewest stops. Because multiple lines may use the same track, a passenger may board an earlier train on a different line, provided it serves their destination or transfer station or an alternate transfer station, if a line to the destination serves it. 


\subsection{Boarding and Alighting}

Because the trains have multiple cars with multiple doors and therefore multiple entry points, passengers are assumed to distribute themselves uniformly along the platform, i.e., arriving passengers are assigned uniformly distributed random locations along the platform. When a train arrives, passengers board the train car nearest their location, provided the train route will convey them to their destination or transfer station. Passengers do not board a train car until all alighting passengers have done so. Passengers board (or alight) at a maximum rate based on the number of doors per car and a maximum of 2 persons per second passing through a doorway. For example, for train cars with 3 double doors, the maximum un/loading rate is 12 per second. Based on the maximum capacity of the train cars, it may take up to 10-15 seconds to (un)load a car. If a train car reaches its maximum capacity, passengers are redirected to other cars. If all train cars are at their maximum capacity, no additional passengers may board. Passengers denied boarding wait at the station for the next train to their destination. This phenomenon occasionally occurs in the simulation (and in reality) during peak operation periods.

For simulations involving a particulate agent where fomite transport may be important, the movements of passengers in the train cars are included in the simulation: after boarding the car, passengers begin searching row by row (two footsteps per row; one row per time step, typically $1 \mathrm{~s})$ through the car looking for an available seat. Passengers boarding through the doorways at either end of the car begin searching toward the center; otherwise, their search direction is chosen by comparing a uniform random number $0 \leq r \leq 1$ with their initial (doorway) location, $x$, relative to the front of the car. If they boarded at the center door, $x=0.5$, they would search forward, if $r \leq 0.5$ and otherwise search rearward. For a train car with four doorways, a passenger boarding at $x=0.33$ or $x=0.67$ would search forward, if $r \leq 0.33$ or $r \leq 0.67$ and otherwise search rearward. Rather than take the first available seat, passengers will continue to search while the next row has the same or fewer occupied seats. In this way, all empty rows are occupied first. Once they encounter a fully occupied row, passengers will stand there unless the next row has fewer standees. If a seat becomes available in their row, e.g., because an alighting passenger has vacated it, a standee will occupy the seat. Upon arriving at a station, passengers alighting at that station stand up, if they are seated, and begin moving toward the nearest doorway, one row per time step. Upon reaching the doorway, alighting passengers are placed on the station platform at the doorway location.

For chemical agents where fomite transport is unimportant, passenger movements and exact locations in the train cars are not included in the simulation, reducing the computation time of the simulation. In these cases, passengers alight on the platform at their destination or transfer station at a random, uniformly distributed position along the length of their train car. (Most train cars have 3-4 double doors, so distributing exiting passengers along the length of the car is not unreasonable.) 


\subsection{Exiting}

For the purpose of tracking passengers as they exit or transfer, each station is divided into four areas: the platform, platform exits (e.g., stairs, escalators, passageways, or turnstiles), mezzanine(s), and mezzanine exits. The platform area is further divided in two separate platforms, with each platform serving one of the tracks, as illustrated in Figure 4 for a generic side-platform station. This also accommodates stations with center express tracks (New York), because express and local trains going in the same direction share a platform, if the station serves express trains. Center-platform stations are also treated as separate platforms for each track because concentrations are computed separately for each track by BGM.

The platforms are further divided lengthwise into zones (approximately 50 feet long). The number of passengers in each zone is counted and combined with the zonal area to determine the passenger number density and then the group walking speed in each zone according to the formula (Society of Fire Protection Engineers [SFPE] 1988, p. 2-108)

$$
S=k(1-a n)
$$

where $S$ is the walking speed $\left(\mathrm{m} \mathrm{s}^{-1}\right), k$ and $a$ are constants $\left(k=1.4 \mathrm{~m} \mathrm{~s}^{-1}, a=0.266 \mathrm{~m}^{2}\right)$, and $n$ is the passenger density (number per square meter). The maximum walking speed, $\sim 1.2 \mathrm{~m} \mathrm{~s}^{-1}$, occurs for $n \leq 0.54$ persons per square meter; to enforce this condition in the above expression, $n$ is replaced by the larger of $n$ or 0.54 . Additionally, a minimum walking speed of $0.1 \mathrm{~m} \mathrm{~s}^{-1}$ is enforced.

After alighting onto the platform, patrons move at the group walking speed for their platform zone toward their optimal platform exit, which is selected as the minimum combination of distance to the exit and length of the exit queue. This exit choice is reevaluated for each passenger at each time step. In this way, if the queue for a particular exit grows too long, passengers will move to a more distant exit with a shorter queue.

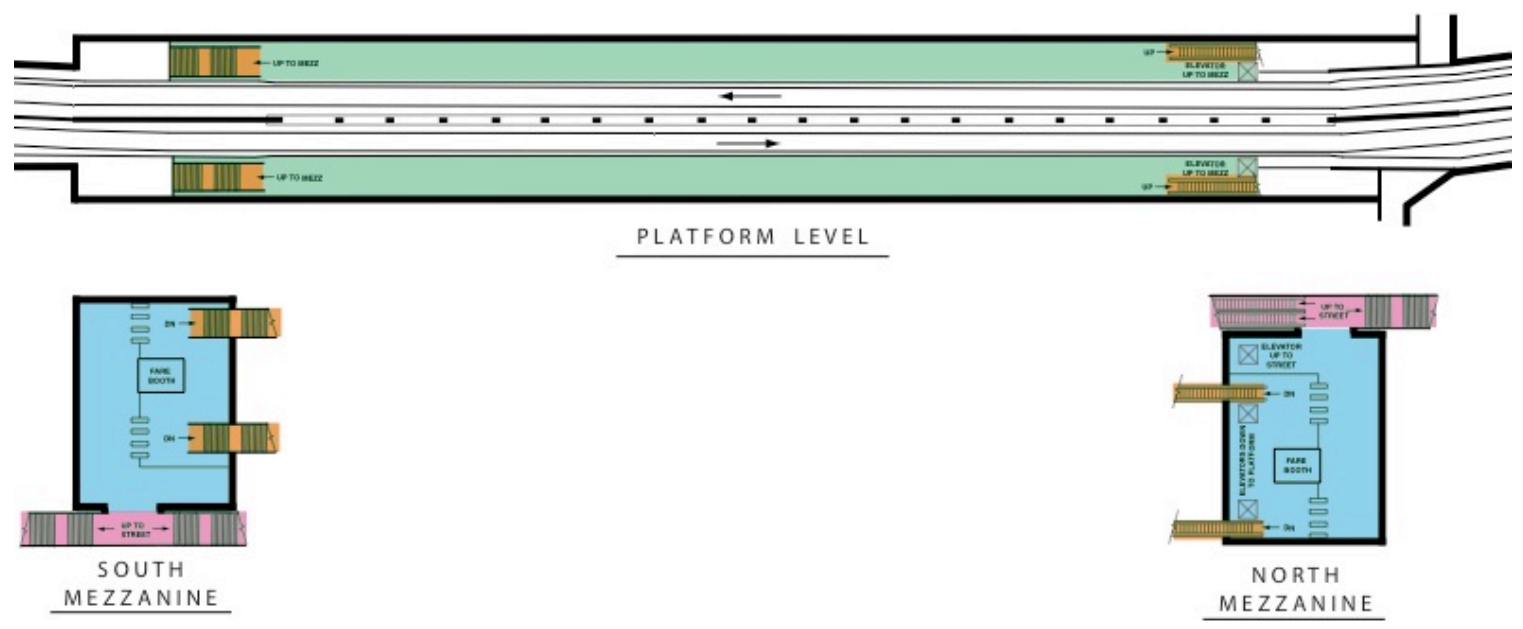

Figure 4. A typical side-platform station showing the platforms (green), platform exits (orange), mezzanines (cyan), and mezzanine exits (magenta). 
Passengers' exposure accumulates according to the concentration at their instantaneous location on the platform. If, during a chemical agent release, passengers' exposure exceeds their incapacitating level, following an agent-dependent delay $(30 \mathrm{~s}-1 \mathrm{~h})$ for the physiological effect of the dosage to be manifested, they are overcome at that point and their locations no longer change. Because these passengers will impede the egress of other passengers, their presence continues to contribute to the number density on the platform and reduces the group walking speed of passengers still trying to exit.

Each station has one or more platform exits, which may serve either a single track for sideplatform stations or both tracks for center-platform stations. Each exit has a queue and several descriptive parameters, including its location along the platform, vertical rise, number of persons that can pass through simultaneously (in parallel), and the mezzanine it leads to (if any). The vertical rise may be zero, in which case the exit is a doorway, passageway, or other choke point such as a platform-level turnstile. Escalators are treated as stairways, assuming that during an evacuation all escalators would be stopped and used as stairs. Group speed up a stairway is calculated based on the number density of people on the stairway, using the same formula given above for the walking speed along the platform, except that $k=1.23$ (SFPE 1988, p. 2-108, Table 2.6-2), which assumes a riser height of 6.5 inches and a tread of 13.0 inches. If the group speed up the stairway is less than the riser height, such that the bottom stair is not cleared after a time step, the passenger(s) at the head of the queue for the stairs are not allowed on the stair and the queue will grow in length. Unlike the platforms, the minimum speed on stairways may be zero, which allows them to become blocked. However, the choice of riser and tread dimensions combined with a width of $1 \mathrm{~m}$ per simultaneous person prevents the number density from becoming high enough to cause the speed to fall to zero, except for chemical agent releases during which people may be overcome on the stairways and stop moving.

Upon exiting the platform (e.g., reaching the top of the stairway or passing through a platformlevel turnstile), transferring passengers are immediately repositioned at a uniformly distributed random location on the cross-line platform where they will await their connecting train. Exiting passengers are either placed on the connecting mezzanine or they exit the subway, if no mezzanine exists. (The "mezzanine" may be the unpaid area beyond the turnstiles at the platform level.) Each mezzanine has a single exit, which, like the platform exits, may accommodate multiple passengers simultaneously. Passengers traverse the specified distance to the mezzanine exit at a group speed calculated, as for the platforms, with a number density based on the specified floor area for the mezzanine. Mezzanine exits are treated identically to platform exits. Exposures on the mezzanine and mezzanine exits are based on the concentration at a specified coordinate along the platform, which is usually the coordinate of the platform exit connecting to the mezzanine or the coordinate centroid for multiple platform exits. This reflects the effect of the pressure change caused by train arrivals to force air from the platform up the stairways/escalators to the mezzanine and out the station exits. 


\section{Exposure and Response}

\subsection{Exposure}

As they transit the system, passengers' individual exposures to airborne agents, $C T$, are calculated as the integrated airborne concentration, $C(t)$, to which they are exposed during their time $T$ in the subway after their arrival at time $t_{0}$

$$
C T=\int_{t_{0}}^{t_{0}+T} C(t) d t
$$

For the purpose of assessing the health consequences of an agent release, the $C T$ values are assumed to be the passengers' inhaled dosages. The BGM exposure model does not include a model of inhalation because the typical particle sizes (and gases) of interest are considered to be fully respirable, i.e., they reach the lungs when inhaled. However, each passenger's final $C T$ value is output at the end of the simulation, should a separate analysis of inhalation be desired.

For biological agents whose viability decreases over time, an adjustment factor, $f_{v}$, is included in the $C T$ calculation

$$
C T=\int_{t_{0}}^{t_{0}+T} C(t) f_{v}(t) d t
$$

where

$$
\begin{gathered}
f_{v}(t)=\frac{1}{\gamma t}\left(1-e^{-\gamma t}\right), \quad 0 \leq t \leq T_{r} \\
f_{v}(t)=\frac{1}{\gamma T_{r}}\left(e^{-\gamma\left(t-T_{r}\right)}-e^{-\gamma t}\right), \quad t>T_{r} .
\end{gathered}
$$

Here $\gamma$ is the viability decay rate, $t$ is the time since the beginning of the release, and $T_{r}$ is the duration of the release.

Depending on the agent characteristics, each passenger is randomly assigned an individual effective dosage level for incapacitation (chemical agent) and/or lethality (chemical/biological agents) based on a standard dose-response curve defined by its probit (Bliss) slope and $E C T_{50}$ values (refer to Appendix C for definitions; a dose-response curve for fatal exposure to anthrax, based on parameters from Foster, Sugiyama, and Nasstrom (2001), is presented in Figure 5.) That is, each passenger is assigned an effective dosage level that would be just sufficient to produce the specified biological response in the fraction of the population indicated by a uniform random number $0 \leq r \leq 1$. For consistency, the same random number is used to assign an individual's incapacitation and lethal dosage levels. If, during the simulation, an individual's exposed dosage reaches or exceeds their effective dosage level, that individual is considered incapacitated or fatally exposed. In this way, the simulated population exhibits the range of sensitivity to agent exposure likely to be experienced by an actual population. 


\section{Bacillus anthracis}

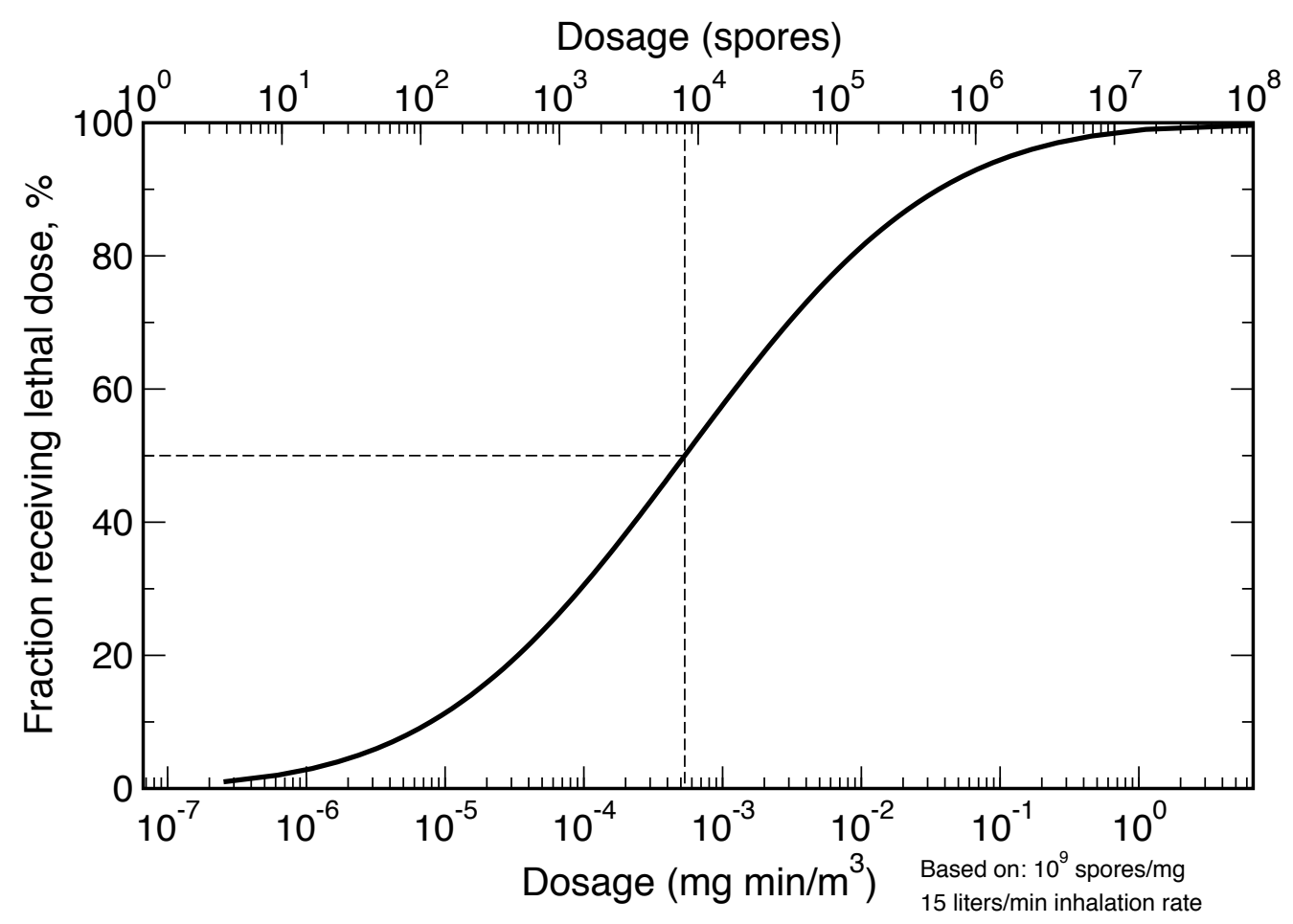

Figure 5. The dose-response curve for lethal exposure to anthrax if untreated. The dashed line indicates the $E C T_{50}=L D_{50}$ dosage, which would be lethal to $50 \%$ of the exposed population.

For (chemical) agents having a toxic load exponent $n>1$, a brief exposure to a large concentration is worse, i.e., causes the specified biological responses in a greater fraction of the population than a long exposure to a low concentration, even though the $C T$ value is the same (ten Berge, Zwart, and Appelman 1986). In this case, passengers' exposure is calculated as

$$
C^{n} T=\int_{t_{0}}^{t_{0}+T} C^{n}(t) d t .
$$

Individual thresholds for incapacitation and lethality are defined using dose-response functions defined by the probit slope, toxic load exponent, and $E C^{n} T_{50}$ values. A more thorough discussion of dose-response models and toxic load is presented in Appendix C.

For chemical agents, in addition to identifying passengers receiving an incapacitating and/or lethal exposure, those passengers exceeding Acute Exposure Guideline Levels (AEGLs) are also identified. These levels are defined as follows (National Research Council 2003):

AEGL-1 The general population, including susceptible individuals, could experience notable discomfort, irritation, or certain asymptomatic nonsensory effects. However, the effects are not disabling and are transient and reversible upon cessation of exposure. 
AEGL-2 The general population, including susceptible individuals, could experience irreversible or other serious, long-lasting adverse health effects or an impaired ability to escape.

AEGL-3 The general population, including susceptible individuals, could experience lifethreatening health effects or death.

Figure 6 presents the number of unexposed (green), less-than-fatally exposed (red), and fatally exposed (black/grey) patrons in a central subway station where passengers may transfer between several lines following a simulated bioagent release at 08:30 in a nearby station. The exposures are presented according to whether the patrons are (1) waiting on the platform for a train, (2) exiting the platform after alighting from a train, (3) on (or queued for, in blue) the stairs/escalators from the platform to the mezzanines, (4) on the mezzanines, and (5) on the mezzanine stairs/escalators. The regular arrival and departure of trains and passengers is evident.

During the morning peak travel period, comparatively few patrons are entering this station, and many patrons waiting on the platform are transferring from one train to another. In particular, the fatally exposed passengers have transferred from trains passing through the release station. That the airborne concentration is less than that required to cause a fatal exposure is evident from the number of patrons waiting and exiting that received less than a fatal dosage, and that the number

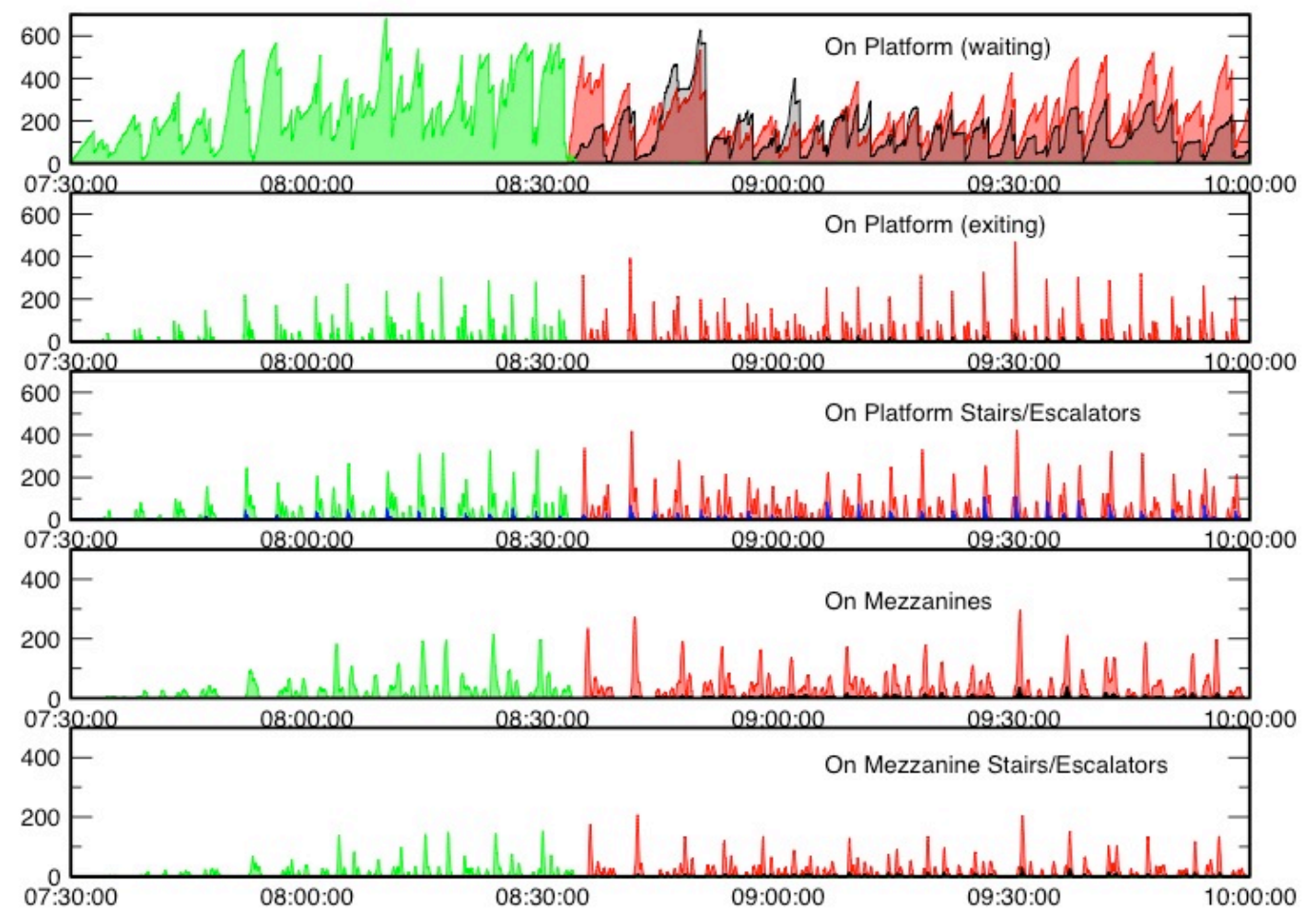

Figure 6. Number of unexposed (green), less-than-fatally exposed (red), and fatally exposed (black) patrons following a bioagent release at 08:30 in a nearby station. The number queued for platform stairs/escalators is shown in blue. 
of fatal exposures is not growing with time. It is also evident that patrons' residence time in the station, and subsequent exposure, is considerably greater while waiting for a train than as they exit.

\subsection{Patron Response}

The BGM passenger exposure model also considers population response to these health effects. As previously discussed, patrons that become incapacitated subsequent to exposure to a chemical agent may impede other patrons' ability to exit, either by reducing the group walking speed in the local area or by partially or completely blocking an exit. Additional patron responses considered in the model include rapid self-evacuation and/or directed evacuation from the subway (as would occur with a chemical detection system), along with the system response of the subway operators.

To evaluate the benefit of chemical detection systems, passengers' response in the absence of detection must be determined first. For instance, chemicals that have a strongly discernable or objectionable odor, or exhibit other prompt effects, will drive subway patrons to self-evacuate. For some chemicals, this response can lead to large-scale self-evacuation and result in shutdown of the subway, as the system operators respond to the evolving chemical incident. Accordingly, patron exposure and behavior are updated and evaluated within BGM for each time step in the simulation, thereby allowing the passengers' response to affect (and be affected by) the subway system operators' response. This integrated modeling framework provides a realistic analysis of detection system benefit over a "no-detection" baseline.

In evaluating patron response, four evacuation triggers are considered:

- $\quad$ Patron is personally affected: When the local agent concentration exceeds a level for which acute effects are present (e.g., runny eyes, tight chest) or a clearly objectionable odor, the patron will self-evacuate.

- $\quad$ Patron observes others affected: The patron witnesses other passengers in distress or overcome on a station platform or in a train car.

- Patron observes others evacuating: The patron witnesses a general exodus of the local area or station.

- Alarm or announcement: This can be in the form of an individual station evacuation or a general system shutdown in response to a detector alarm.

These trigger mechanisms are implemented as follows. When the local agent concentration exceeds a specified acute effects level, e.g., odor for a sustained period - currently 2 minutes - a patron will decide to self-evacuate. The delay represents the period of time the effect(s) must persist before the patron determines that self-evacuation is necessary. Once patrons decide to evacuate while waiting for a train, they seek the nearest exit. When patrons decide to evacuate while on a train, they alight at the next stop and exit there. 
When at least twenty patrons at a station are evacuating because of odor or other acute effects, all patrons in the station or train car decide to evacuate. The threshold of twenty patrons was chosen by consensus with modeling groups at Sandia National Laboratory and Defence Science and Technology Laboratory (U.K.) so that more than a few patrons evacuating would be required to precipitate a general exodus but not so many that sparsely populated stations or train cars would not self-evacuate. The actual threshold probably varies from individual to individual depending on their perception of risk as well as on the context, e.g., whether the odor was strong or faint, whether suspicious persons or activities were observed, etc. In the absence of observational data on self-evacuation to determine the person-to-person variation in the threshold, a single constant value was implemented.

When at least three patrons in the vicinity of each other $( \pm \sim 30 \mathrm{~m})$ at a station are incapacitated, all patrons at that station begin to self-evacuate. A minimum of three incapacitated patrons are required to initiate self-evacuation because a single person could be incapacitated by a medical issue (e.g., a heart attack) and two persons could be involved in an altercation, but three or more persons on the floor potentially indicates a generally hazardous situation. The vicinity restriction establishes that the incapacitations likely stem from the same cause and that the incapacitated patrons are simultaneously visible to other patrons on the platform, which is necessary to trigger a general self-evacuation. For train cars, no vicinity restriction is necessary due to their sizewhen three or more passengers in a train car are incapacitated, all passengers in that car decide to self-evacuate at the next stop.

Once all of the patrons at a station are evacuating, no new arrivals occur, because arriving patrons witness the general exodus and choose not to enter. This is the case for both self-directed evacuation and evacuation directed by an alarm or announcement.

\subsection{System Response}

A variety of system response options may be (and have been) investigated using BGM to assess their efficacy as part of a subway's concept-of-operations, or "ConOps," procedures for a chemical incident; a detailed discussion of these procedures is beyond the scope of this report. However, within the context of the simulation, train operations may be altered in response to either self-evacuation or detector alarms, including reducing train speed, reversing train direction, skipping stations, or stopping train operations altogether for the purpose of minimizing patron exposures.

As an illustration of patron and system response in prompt chemical attack scenarios, Figure 7 presents the AEGL exposures and fatalities at a subway station in the central business district of a fictitious subway system following the release of sarin gas (GB). It takes approximately 8 minutes for the sarin concentrations to rise to the point where patrons are incapacitated and self-evacuation initiates. A little more than 10 minutes after the release begins, the Operations Control Center personnel recognize a major event. The decision to declare a chemical emergency is made 14 minutes after the release begins, and the entire system is evacuated over the next 10-20 minutes. The AEGL exposures level off after this decision is made because exposed patrons evacuate and unexposed patrons are prevented from entering the 
contaminated area. However, in this example, fatalities continue to increase as patrons evacuate and incapacitated individuals that cannot self-evacuate accumulate a fatal dosage.

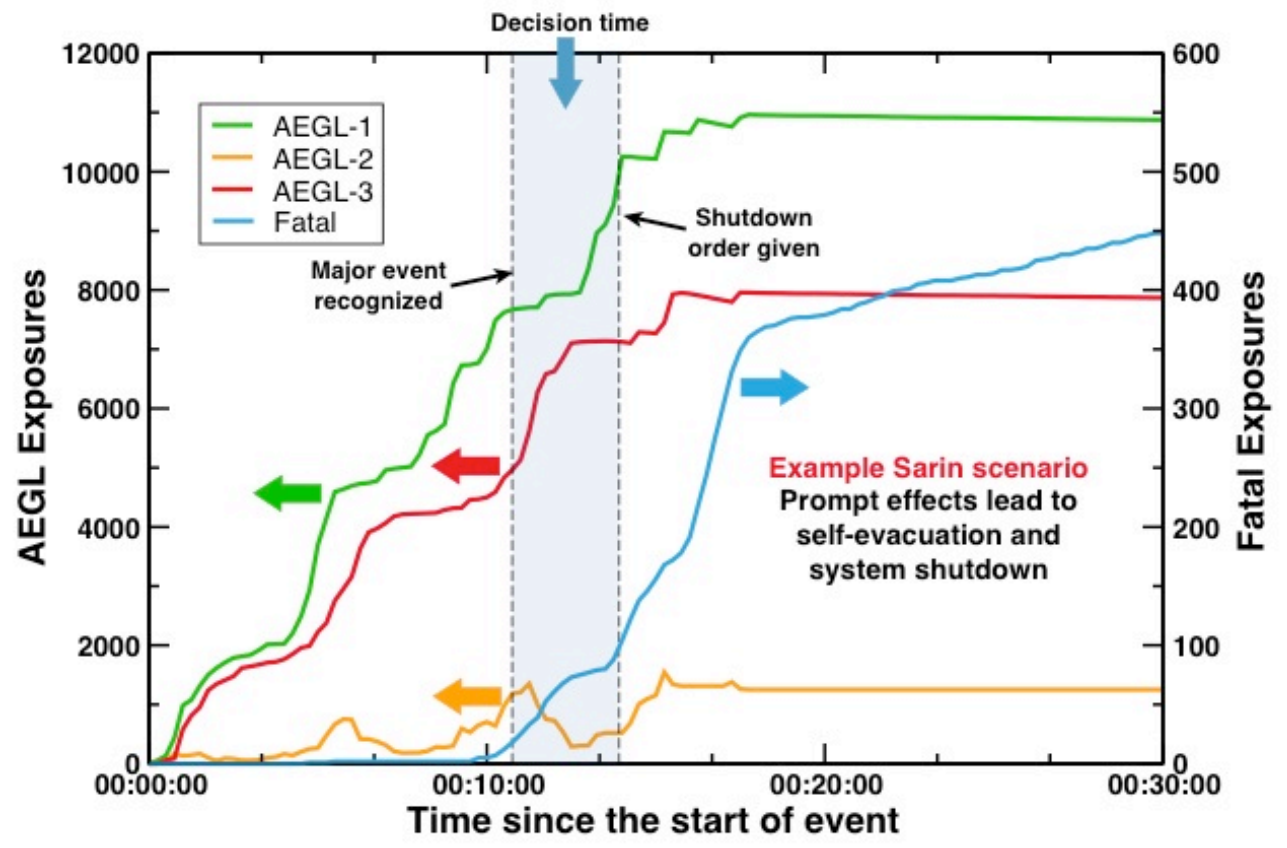

Figure 7. AEGL exposures (left axis) and fatal exposures (right axis) at a station in the central business district following the simulated release of sarin (GB) to illustrate the mitigating effects of self-evacuation and system shutdown responses. 


\section{Fomite Transport}

Particulate agents may be deposited on subway patrons while waiting for a train on a station platform or while riding in a train car. These particles may subsequently be shed (resuspended) from these patrons in different stations, or even beyond the subway. In effect, passengers serve as fomites (carriers) of bioagent particles, representing a transport mechanism distinct from the subway train-induced airflows and train cars. In addition, bio-particles deposited on station or train car floors may be resuspended by the action of passengers' footsteps, or they may adhere to the passengers' shoes and be re-deposited elsewhere.

\subsection{Particle Deposition and Resuspension}

The net rate of deposition of particle mass, $m_{p}$, onto each subway patron is modeled as the balance of deposition and resuspension

$$
\frac{d m_{p}}{d t}=\left(v_{d} C A_{p}-r_{p} m_{p}\right) f
$$

where $v_{d}$ is the deposition velocity, $C$ is the airborne mass concentration in the tunnel or station segment or train car, $A_{p}$ is the surface area of a patron, and $r_{p}$ is the normalized resuspension rate (the fraction of deposited mass resuspended per unit time) for particles shed from a patron's clothing. The factor $f$ represents the fraction of the patron's surface area available for deposition and resuspension. For patrons that are standing or walking, $f=1$; for seated passengers, $f=0.5$.

To account for variations in subway patrons' clothing types (e.g., cotton, wool), each patron is assigned a deposition velocity drawn at random from a lognormal distribution having a median of $36 \mathrm{~m} \mathrm{hr}^{-1}$ and a geometric standard deviation of 2.7, which were determined from measurements of particle deposition onto people conducted in an actual transit station (Liljegren, et al. 2016).

The resuspension rate for patrons that are walking (boarding or alighting from a train, or exiting a station) is larger than for passengers that are stationary (standing or seated). All walking patrons have the same resuspension rate, and all stationary patrons have the same resuspension rate; however, the walking and stationary resuspension rates vary with particle size and other factors. Resuspension rates are discussed in detail in Appendix D.

In addition to resuspension ("shedding") from patrons' clothing, particles previously deposited on train car floors may be resuspended by the action of patrons' footsteps as they board or alight. In this case, the rate of mass resuspension from the floor by a single patron is

$$
\frac{d m_{f}}{d t}=r_{f} \frac{m_{f}}{A_{f}} A_{s} N_{s}
$$


where $m_{f}$ is the mass of particles deposited on the train car floor; $A_{f}$ and $A_{s}$ are the surface areas of the floor and a footstep, respectively; $N_{s}$ is the number of footsteps the patron takes; and $r_{f}$ is the resuspension rate from flooring. $A_{s}=0.03 \mathrm{~m}^{2}\left(0.3 \mathrm{ft}^{2}\right)$ for all patrons.

Mass conservation requires that the rate of change in the airborne concentration for a stationary patron be described by

$$
\frac{d C}{d t}=\left(-v_{d} C \frac{A_{p}}{V}+\frac{r_{p} m_{p}}{V}\right) f
$$

where $V$ is the volume of the station segment or train car. For a walking patron,

$$
\frac{d C}{d t}=-v_{d} C \frac{A_{p}}{V}+\frac{r_{p} m_{p}}{V}+\frac{r_{f} m_{f}}{V} \frac{A_{s} N_{s}}{A_{f}} .
$$

The first term on the right-hand side represents a loss of airborne particles by deposition onto patrons, because a positive deposition velocity indicates particle movement from the air toward the depositing surface. For stationary patrons, the last term on the right-hand side is zero because $N_{s}=0$.

To illustrate the significance of fomite transport, Figure 8 presents the integrated concentration or dosage along a subway line for a gas and for particles ranging in size from $1 \mu \mathrm{m}$ to $10 \mu \mathrm{m}$ with and without fomite transport (i.e., with and without particle deposition to and resuspension from passengers). Without fomite transport, the particle concentration, and the corresponding dosage, are depleted solely by deposition caused by gravitational settling, which increases with particle size. The effect of deposition onto passengers is to reduce the airborne concentration near the release location; that is, passengers initially represent a removal mechanism, with larger

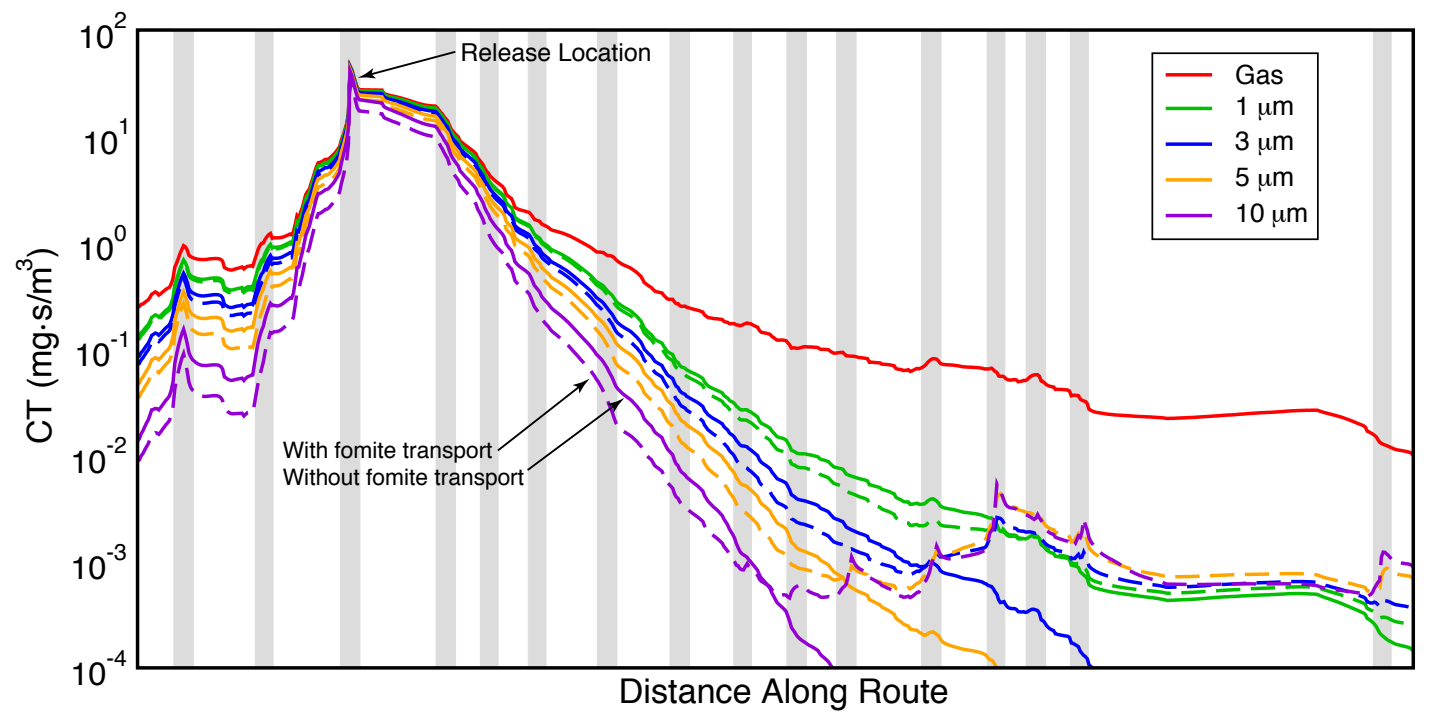

Figure 8. Dosage along a subway line for a gas and for different size particles without (solid lines) and with (dashed lines) fomite transport. The gray bars indicate station platform locations. 
particles experiencing greater deposition. However, as contaminated passengers travel away from the release location, they represent a source of particles for resuspension, which enhances the airborne concentration and increases the dosage at distant stations (and in train cars).

\subsection{Contact Transfer}

Contact transfer is the process whereby particles are transferred from one surface to another when the two surfaces come into contact, such as the transfer of particles deposited on a subway patron's clothing to a train car seat when they sit down, then a subsequent transfer to another patron's clothing when they later occupy the same seat. The transfer of particle mass by contact between a patron's clothing and train car seat surfaces is modeled as

$$
m=f \rho A_{\text {contact }}
$$

where $m$ is the mass transferred, either from patron to seat or seat to patron; $f$ is the fraction transferred by contact, $\rho$ is the mass loading per unit area of the surface from which particles are transferred, and $A_{\text {contact }}$ is the contact area between the two surfaces.

Because it is not computationally feasible to calculate and track the mass deposited on each seat, the seat mass loading represents an average, based on the deposited mass in the train car and the train car surface area, whereas the mass loading for each passenger is calculated using the mass deposited on each passenger. The contact area is the sum of the contact area for the seat bottom $\left(0.16 \mathrm{~m}^{2}\right)$ and the seat back $\left(0.2 \mathrm{~m}^{2}\right)$.

McDonagh, Sextro, and Byrne (2012) have investigated the contact transfer between cotton, fleece, brass, and plastic laminate surfaces. They found that varying contact time or particle loading had a minimal effect on the measured transfer fraction. For low contact pressures, they measured high transfer fractions from hard surfaces - brass and plastic laminate - to fleece (41\% and $30 \%$, respectively) and a low transfer fraction from fleece to laminate $(2 \%)$; however, laminate-to-cotton transfer (3\%) was less than cotton-to-laminate $(7 \%)$, although brass-to-cotton transfer was higher (11\%), and cotton-to-cotton (3\%) and laminate-to-laminate transfer (3\%) were comparable.

They measured higher transfer fractions for contact pressures above a threshold pressure, which were generally more than double those for low contact pressures. For cotton-to-cotton transfer (which was comparable to laminate-to-cotton transfer at low contact pressures), they measured an average transfer fraction of $8 \%$ for contact pressures above $3500 \mathrm{~Pa}$. The seat bottom contact area of $0.16 \mathrm{~m}^{2}$ corresponds to a $57 \mathrm{~kg}$ person, which is below the $5^{\text {th }}$ percentile weight for adult men and near the $15^{\text {th }}$ percentile weight for adult women (McDowell et al. 2008). However, the contact pressure on the seat back will be less than this threshold value, corresponding to a 3\% transfer fraction. Weighting these by the contact areas for the seat bottom and seat back, $f_{\text {seat-to- }}$ patron $=0.05$. Although cotton-to-laminate measurements were not carried out at high contact pressures, if the trend of more than doubling the low pressure results holds, then a value of $\sim 15 \%$ may be assumed, and $f_{\text {patron-to-seat }}=0.10$ is obtained. 


\subsection{Particle Tracking}

Particle tracking relies on contact transfer between the floor and peoples' shoe soles to transport particles from one location to another as they walk. Particle tracking is modeled following the approach of Sippola, Sextro, and Thatcher (2014). Based on their laboratory studies, they proposed that the fraction of particles transferred from floor to shoe sole by a single step is approximately constant, but the fraction transferred from shoe sole to floor decreases with each step as particles with less adherence are depleted. They model the shoe-to-floor transfer as

$$
f_{d, j}=f_{d, 12}+\frac{f_{d, 1}-f_{d, 12}}{j}
$$

where $f_{d, j}$ is the downlay (shoe-to-floor) fraction resulting from the $j^{\text {th }}$ step after an uptake (floorto-shoe) transfer step, and $f_{d, 1}$ and $f_{d, 12}$ are, respectively, their measured downlay fractions from the first and twelfth steps following an uptake step. This means that, whereas the uptake fraction is independent of prior steps, the downlay faction depends on the prior history (with an asymptotic limit of $f_{d, 12}$ ). The mass transferred from floor to shoe by a single step is

$$
S=f_{\text {uptake }} \frac{M_{\text {floor }}}{A_{\text {floor }}} A_{\text {shoe }}
$$

where $M_{\text {floor }}$ is the mass of particles on the floor, and $A_{\text {floor }}$ and $A_{\text {shoe }}$ are, respectively, the areas of the floor and shoe sole. The mass on a shoe after the $n^{\text {th }}$ step is the sum of the contributions from all steps

where

$$
M_{\text {shoe }, n}=\sum_{i=0}^{n-1} m_{n-i}
$$

$$
m_{n-i}=S_{n-i} \prod_{j=0}^{i} a_{d, j}
$$

is the mass remaining from the $n-i^{\text {th }}$ step, $S_{n-i}$ is the mass taken up by the $n-i^{\text {th }}$ step, and $a_{d, j}=$ $1-f_{d, j}$ is the fraction of the particle mass remaining on the shoe following the $j^{\text {th }}$ step after it was taken up from the floor $\left(a_{d, 0}=1\right)$. The mass on the floor after the $n^{\text {th }}$ step is simply

$$
M_{\text {floor }, n}=M_{\text {floor }, n-1}-\left(M_{\text {shoe }, n}-M_{\text {shoe }, n-1}\right) \text {. }
$$

The computational cost associated with retaining the history of each subway patron's steps as well as summing their contributions to each current step is prohibitive. Recognizing that the downlay fraction approaches an asymptote after $n_{\max }$ steps $\left(n_{\max }=12\right)$, the mass on a shoe after the $n^{\text {th }}$ step is 


$$
M_{\text {shoe }, n}=\sum_{i=0}^{n_{\max }-1} m_{n-i}+\sum_{i=n_{\max }}^{n-1} m_{n-i}
$$

which only requires retaining the history of the previous $n_{\max }$ steps. That is, because $a_{d, n_{\max }}=$ constant for $n>n_{\max }+1$,

$$
\sum_{i=n_{\max }}^{n-1} m_{n-i}=m_{n-n_{\max }}+a_{d, n_{\max }} m_{(n-1)-n_{\max }}
$$

In their laboratory studies of tracking, Sippola et al. (2014) varied the flooring type, shoe type, particle size, initial loading, and step pressure. They found that dress shoes with smooth soles took up and laid down more particles than athletic shoes with aggressive tread patterns, possibly because the actual contact area for smooth soles is greater. They also found that the fraction transferred either from floor to shoe or shoe to floor depended more significantly on particle size (5-10 $\mu \mathrm{m}$ particles exhibited higher transfer rates than 1-4 $\mu \mathrm{m}$ particles) than on contact pressure, i.e., on a person's weight; their results revealed no dependence on the initial mass loading of the floor. Accordingly, each patron in the simulation is assigned uptake and downlay parameters based on the particle size for either a dress shoe or athletic shoe with a contact area of $200 \mathrm{~cm}^{2} \mathrm{~m}^{-1}$; patrons' weight is not considered. Hard (non-carpeted) flooring is assumed. Patrons are assumed to take 2 steps per second, which is consistent with an observed comfortable walking pace of 108-118 steps per minute for adults (Perry 1992, p. 455, Table 21.2); this implies that people walk more slowly or more quickly by shortening or lengthening their stride.

Passengers entering a contaminated station track material throughout the system. To initialize the amount of material on the shoes of entering patrons, a station entrance is randomly selected for each person as they enter, and the mass transferred between the platform and their shoes is calculated as they walk to their initial location along the platform. Then, material is transferred between train car floors and patrons' shoes as they board and alight from the trains, and finally as they exit the subway.

The effect of tracking is illustrated in Figure 9, which presents the amount of material deposited along southbound station platforms and tracks without and with particle tracking. Without tracking, deposition is caused primarily by gravitational settling, whereas tracking significantly 


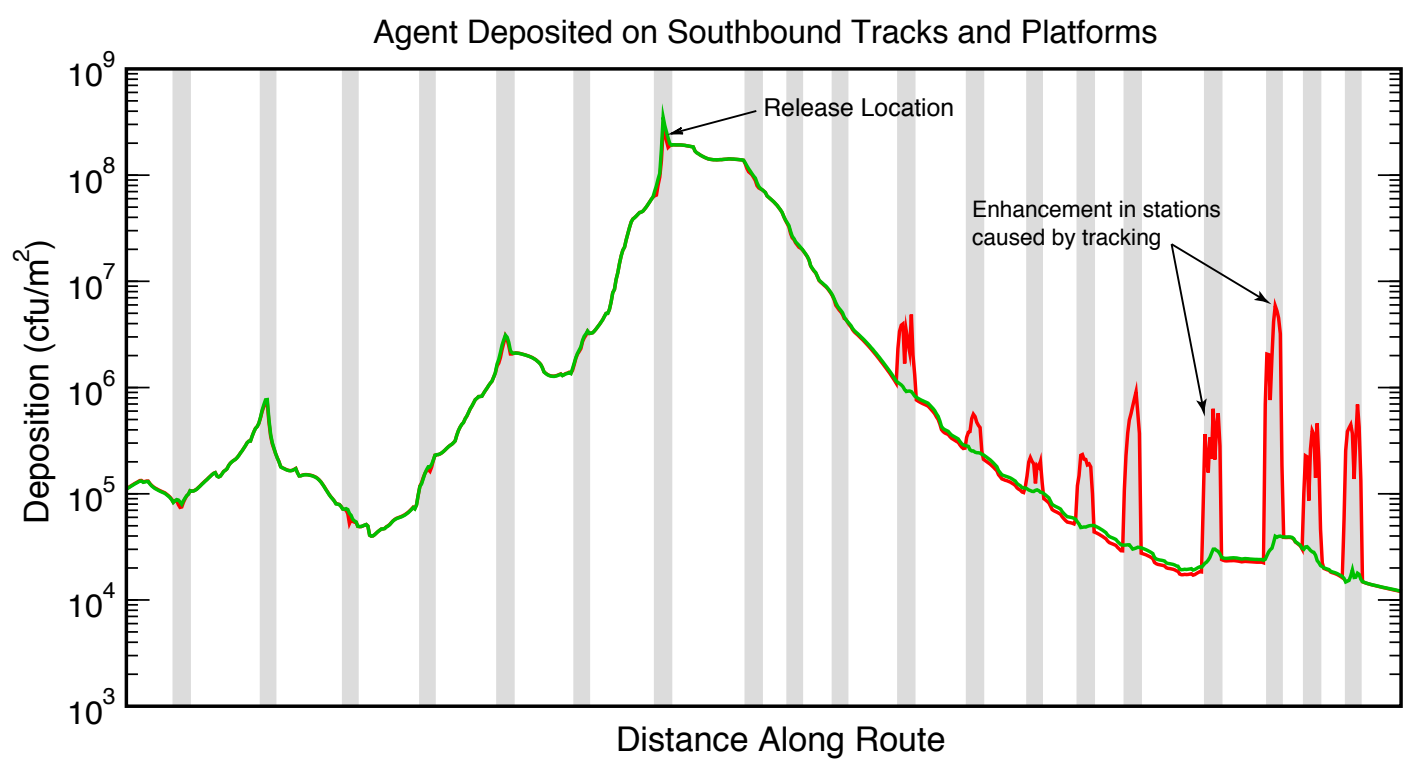

Figure 9. Number of colony-forming units (cfu) deposited per unit area on the southbound platforms and tracks without (green) and with (red) particle tracking. The gray bars indicate station platform locations.

enhances the amount of material deposited on southbound platforms for stations south of the release location. Similarly, tracking enhances the amount of material deposited on northbound platforms of stations north of the release location (not shown). Variations in the amount deposited by tracking are related to the number of patrons alighting at that station.

\subsection{Gas Adsorption and Desorption}

The sorption of gaseous agents to/from subway patrons' clothing is modeled using a 2-sink approach (Singer et al. 2005, 2007) identical to the approach for sorption with subway surfaces. The 2-sink model formulation and the rate constants used in BGM are described in Part I.

In general, sorption to passengers' clothing is not a significant mechanism for agent transport in the subway. Because subway passengers' residence time in any station is limited to a few minutes while waiting for a train or while exiting from the station, the amount of agent that may be adsorbed in a station with high concentration and desorbed in a train car or station with low concentration is also limited. Nevertheless, in the Tokyo sarin attack, sorption proved to be an issue for secondary contamination of ambulances and hospitals where large numbers of mildly contaminated patrons were concentrated into relatively small spaces. However, in that case, opening windows and improving the ventilation of the vehicles and rooms caused the symptoms to rapidly abate (Smithson and Levy 2000, Chapter 3, p. 99). 


\section{Summary}

The population model developed for the Argonne Below Ground Model accounts for the timing and movement of individual subway patrons as they travel through the system. When available, actual turnstile data is used to determine the time and station of entry for each passenger as well as their destination station, employing standard techniques for treating the random process that governs their exact arrival times. If turnstile data is not available, algorithms that reproduce demonstrated trends have been developed to estimate timing and origin-destination pairing.

Using airborne agent concentrations calculated by BGM, the population model calculates each person's exposure along their journey, referencing their individual effective incapacitation and lethal exposure levels to determine the consequences of their exposure. Individual effective levels are determined using published values for the probit slope, median effective level, toxic load exponent, and viability decay rate for the specific agent being considered.

For chemical agents, subway patrons may self-evacuate based on their individual and group responses to the particular material released. These responses may, in turn, result in a subway system response, such as single station evacuation, partial or full system shutdown, skipping stations, etc. This capability allows a baseline to be established for assessing the efficacy of proposed response strategies or detection systems, as well as providing a more complete basis for evaluating countermeasures or response options in realistic release scenarios.

The population model also accounts for fomite transport, i.e., the transport of particulate (biological) agents by contaminated individuals through (1) the resuspension of material deposited on their clothing, (2) resuspension by patrons' foot traffic of material deposited on the station and train car floors, (3) tracking of deposited material on patrons' shoes, and (4) the transfer of material between patrons' clothing and train car seats when they contact. These mechanisms are modeled based on specific, though limited, laboratory studies of the individual phenomena, but have not been confirmed by experiments in actual venues. As a result, including these effects in a simulation is a user-selected option.

The number of patrons exhibiting a specific health consequence (e.g., reversible, irreversible, or fatal) is a critical metric for subsequent optimization analysis of, for example, detector performance specifications, detection architecture designs, and system response strategies. Unlike the BGM transport and dispersion model, however, which has been validated through extensive measurements of gas and particle tracers released in actual subways, no validation measurements of the population model are available. Nevertheless, we believe the results are plausible and generally agree with expectations although they occasionally yield counterintuitive insights. 
This page intentionally left blank. 


\section{Works Cited}

Bliss, C. I. (1934). The method of probits. Science, 76, 38-39.

Brown, D. F., and Liljegren, J. C. Argonne Below Ground Model Part I: transport and dispersion in underground transportation systems. Technical Report ANL/DIS-14/12, Argonne National Laboratory.

Foster, K. T., Sugiyama, G. A., and Nasstrom, J. S. (2001). LLNL's chemical and biological source term dispersion modeling database for the CBNP M\&P thrust area. Technical Report UCRL-ID-143281, Lawrence Livermore National Laboratory.

Freihaut, J. D., Bahnfleth, W. P., and Hu, B. (2008). Phase 2 Task 4 Report: Quantification of floor vibration and aerodynamic swirl effects on particle resuspension properties. Pennsylvania State University, Department of Architectural Engineering.

Hession, H., Byrne, M., Cleary, S., Andersson, K. G., and Roed, J. (2006). Measurement of contaminant removal from skin using a portable fluorescence scanning system. Journal of Environmental Radioactivity, 85, 196-204.

Liljegren, J. C., Brown, D. F., Lunden, M. M., and Silcott, D. (2016). Particle deposition onto people in a transit venue. Health Security, 14(4), 237-249.

Loosmore, G. A. (2003). Evaluation and development of models for resuspension of aerosols at short times after deposition. Atmospheric Environment, 37, 639-647.

McDonagh, A., and Byrne, M. A. (2014a). A study of the size distribution of aerosol particles resuspended from clothing surfaces. Journal of Aerosol Science, 75, 94-103.

McDonagh, A., and Byrne, M. A. (2014b). The influence of human physical activity and contaminated clothing type on particle resuspension. Journal of Environmental Radioactivity, 127, 119-126.

McDonagh, A., Sextro, R. G., and Byrne, M. A. (2012). Mass transport of deposited particles by surface-to-surface contact. Journal of Hazardous Materials, 227, 370-377.

McDowell, M. A., Fryar, C. D., Ogden, C. L., and Flegal, K. M. (2008, October 22). Anthropometric reference data for children and adults: United States, 2003-2006. Retrieved June 6, 2014, from Centers for Disease Control and Prevention, National Health Statistics Reports, Number 10: http:/www.cdc.gov/nchs/products/nhsr.htm

National Research Council. (2003). Acute exposure guideline levels for selected airborne chemicals volume 3. Washington, DC: National Academies Press.

Papoulis, A. (1984). Probability, random variables, and stochastic processes, 2 nd edition. New York, NY: McGraw-Hill.

Perry, J. (1992). Gait analysis: normal and pathological function. Thorofare, NJ: SLACK, Inc. 
Qian, J., and Ferro, A. R. (2008). Resuspension of dust particles in a chamber and associtated environmental factors. Aerosol Science \& Technology, 42, 566-578.

Singer, B. C., Hodgson, A. T., Destaillats, H., Hotchi, T., Rezvan, K. L., and Sextro, R. G. (2005). Indoor sorption of surrogates for sarin and related nerve agents. Environmental Science \& Technology, 39, 3203-3214.

Singer, B. C., Hodgson, A. T., Hotchi, T., Ming, K. Y., Sextro, R. G., Wood, E. E., et al. (2007). Sorption of organic gases in residential rooms. Atmospheric Environment, 41, 3251-3265.

Sippola, M. R., Sextro, R. G., and Thatcher, T. L. (2014). Measurements and modeling of deposited particle transport by foot traffic indoors. Environmental Science \& Technology, 48, 3800-3807.

Smithson, A., and Levy, L.-A. (2000). Ataxia: the chemical and biological terrorism threat and the US response. Retrieved April 30, 2014, from Henry L. Stimson Center, Report 35:

http://www.stimson.org/books-reports/ataxia-the-chemical-and-biological-terrorism-threat-andthe-us-response/

SFPE (Society of Fire Protection Engineers). (1988). The SFPE Handbook of Fire Protection Engineering. Quincy, MA: National Fire Protection Association.

ten Berge, W. F., Zwart, A., and Appelman, L. M. (1986). Concentration-time mortality response relationship of irritant and systemically acting vapours. Journal of Hazardous Materials, 13, 301-309.

Thatcher, T. L., and Layton, D. W. (1995). Deposition, resuspension, and penetration of particles within a residence. Atmospheric Environment, 29, 1487-1497. 


\section{Appendix A: Analysis of NYCTA and PATH Turnstile Data}

The passenger models for both the NYCTA and PATH subways utilize average hourly entry and exit counts derived for each station from one year of turnstile data available online (http://mta.info/developers/access.html). The online data include patron entry and exit counts from each turnstile by fare control area (one or more fare control areas per station) at approximately 4-hour intervals. The timing of the 4-hour reporting intervals is not synchronized, which means that for stations with many turnstiles, entry and exit counts are reported from some turnstiles each hour, whereas for stations with few turnstiles, counts may only be available every 4 hours. To obtain hourly average entry and exit counts, the raw turnstile data were first aggregated over all fare control areas in each station, then normalized by the total reporting time to give the average number per turnstile per hour for each hour reported; these were multiplied by the number of turnstiles in each station to give the hourly entries and exit per station. To fill in gaps and to smoothen discontinuities arising from the 4-hour reporting intervals, the data for each station were then fitted to a Fourier series with 24-hour, 12-hour, and 8-hour modes:

$$
\widehat{N}(t)=a+\sum_{i=1}^{3} b_{i} \sin \left(\frac{2 \pi t i}{24}\right)+c_{i} \cos \left(\frac{2 \pi t i}{24}\right)
$$

The fitted results were normalized to ensure that the total daily entries and exits matched the turnstile data. Figure 10 and Figure 11 illustrate the application of this procedure for two stations: Flatbush Avenue, which is a terminus (end-of-line) station, and Fulton-BroadwayNassau, which is a central station. The trends exhibited by these stations are very similar to those exhibited by the WMATA stations presented in Figure 1: for the terminus station, entries peak in the morning and exits peak in the evening, whereas for the central station exits peak in the morning and entries peak in the evening. Additionally, Figure 12 presents the system-wide hourly weekday entry and exit trends for the New York City subway. These trends are also similar to those exhibited in Figure 2 for the WMATA system; in particular, the entries lead the exits. 


\section{FLATBUSH AV}
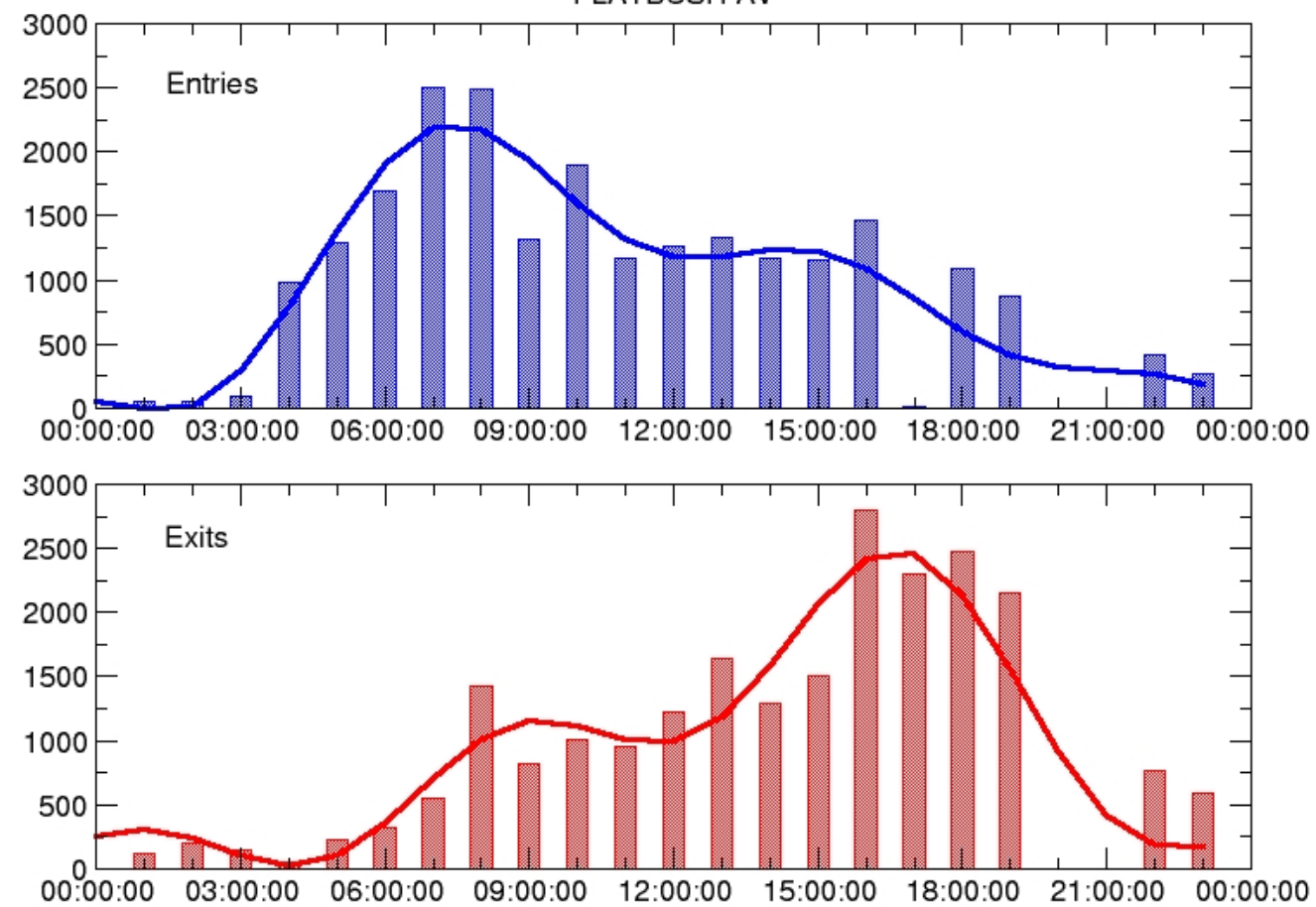

Figure 10. Passenger entries and exits for the Flatbush Avenue Station in Brooklyn. The bars represent the processed turnstile data and the lines are the fitted Fourier series used within BGM.

\section{FULTON/BROADWAY/NASSAU}
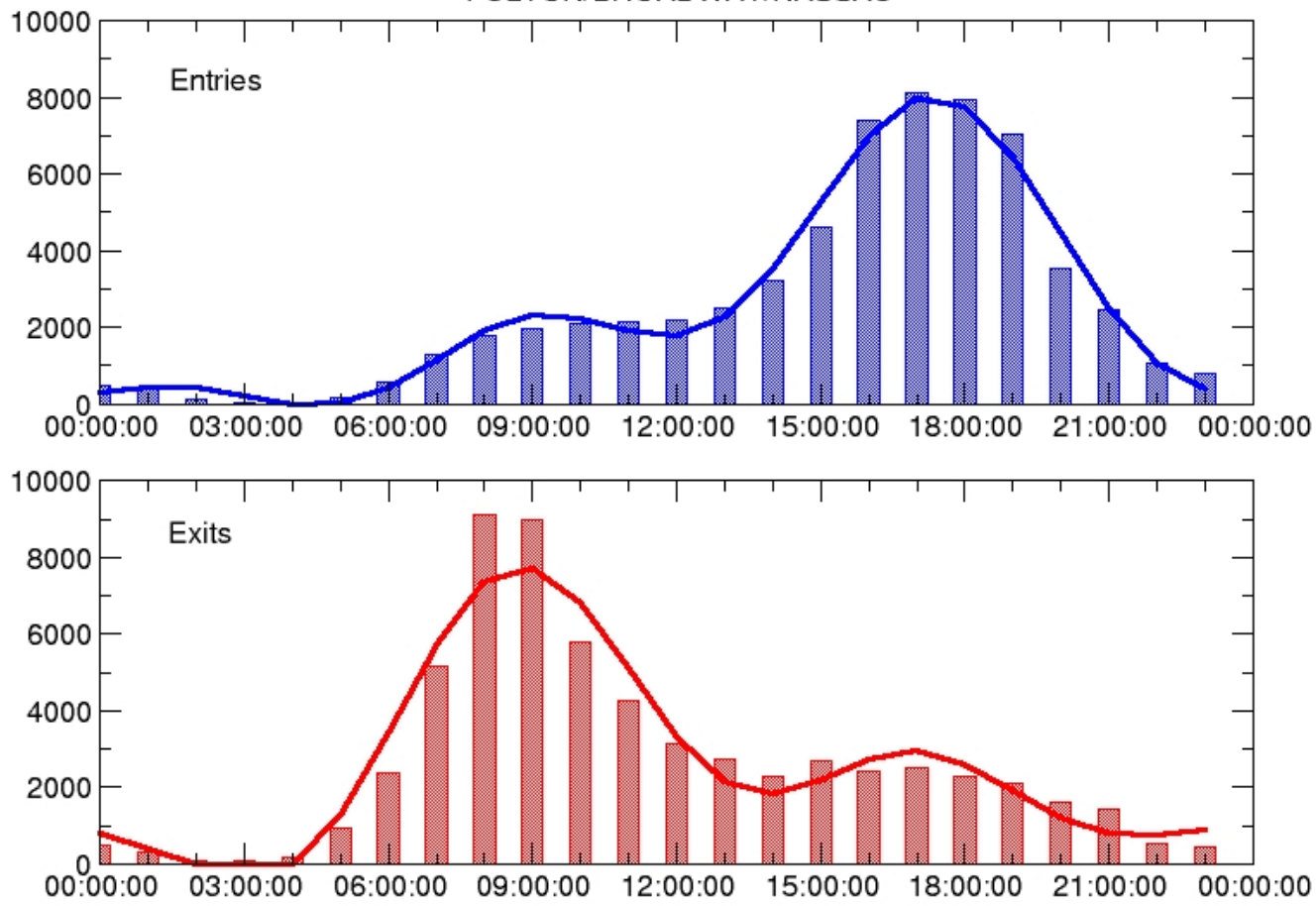

Figure 11. Passenger entries and exits for the Fulton Street Station (formerly Broadway-Nassau) in lower Manhattan. 
New York City Subway Average Hourly Ridership 2011

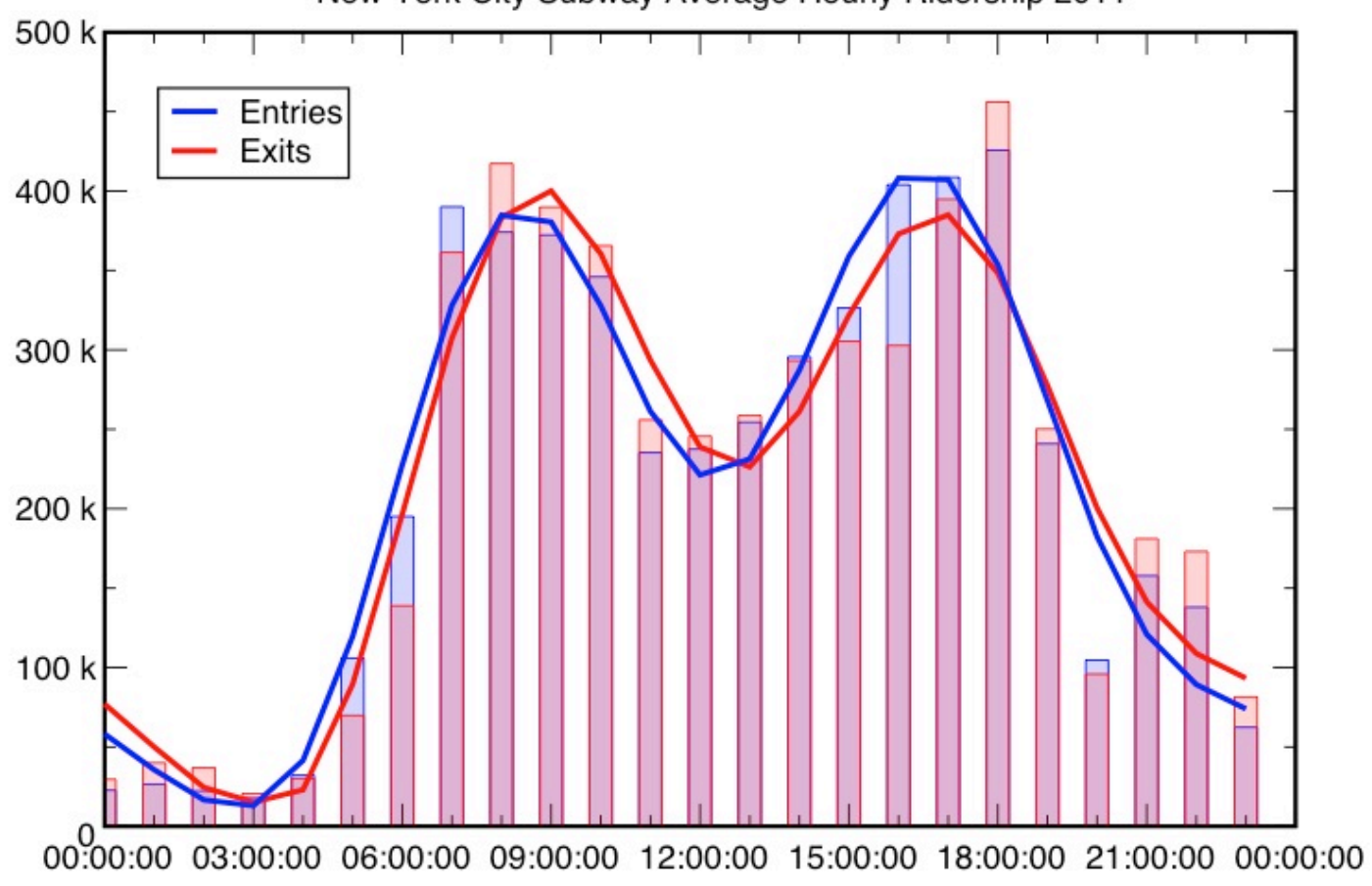

Figure 12. Average hourly weekday entries and exits for the New York City subway (2011 turnstile data). The bars represent the processed turnstile data and the lines are the fitted Fourier series. 
This page intentionally left blank. 


\section{Appendix B: Calculating Origin-Destination Pairing}

\section{B.1 A Single-Line Subway (No Transfer Stations)}

For a subway comprising a single line, the probability of exiting at the $i^{\text {th }}$ station on line A is

$$
P\left(i_{A}\right)=\frac{N_{i_{A}}}{N_{A}}
$$

where $N_{i_{A}}$ is the number of people that exit at station $i_{A}, N_{A}=\sum_{i=1}^{n_{A}} N_{i_{A}}$ is the number of people exiting at all stations on line $\mathrm{A}$ and $n_{A}$ is the number of stations on line A. The probability of exiting at the $i^{\text {th }}$ station on line A having entered at the $j^{\text {th }}$ station on line $\mathrm{A}$ is

$$
P\left(i_{A} \mid j_{A}\right)=\frac{N_{i_{A}}}{N_{A}-N_{j_{A}}}
$$

assuming people do not exit at the same station they entered. Then the number of people exiting at the $i^{\text {th }}$ station that entered at the $j^{\text {th }}$ station is simply

$$
N_{i_{A} j_{A}}=N_{j_{A}} P\left(i_{A} \mid j_{A}\right)
$$

\section{B.2 A Two-Line Subway (One or More Transfer Stations)}

For a subway comprising two lines, A and B, that have one (or more) transfer stations, i.e., stations serving both lines where passengers may transfer between lines,

$$
P\left(i_{A}\right)=\frac{N_{i_{A}}}{N_{A}+N_{A B}}
$$

where $N_{A B}$ is the number of people transferring from line A to line B (which may be a summation over multiple transfer stations). The probability of exiting at the $i^{\text {th }}$ station on line $\mathrm{A}$ having entered at the $j^{\text {th }}$ station on line A (no transfer) is

$$
P\left(i_{A} \mid j_{A}\right)=\frac{N_{i_{A}}}{N_{A}+N_{A B}-N_{j_{A}}}=\frac{N_{i_{A}}}{N_{A}-N_{j_{A}}}\left(1-P\left(A B \mid j_{A}\right)\right),
$$

where $P\left(A B \mid j_{A}\right)$ is the probability of transferring from line A to line $\mathrm{B}$ for passengers entering at the $j^{\text {th }}$ station on line $\mathrm{A}$

$$
P\left(A B \mid j_{A}\right)=\frac{N_{A B}}{N_{A}+N_{A B}-N_{j_{A}}} \cong \frac{N_{A B}}{N_{A}+N_{A B}}=P(A B)
$$


and $P(A B)$ is the probability of transferring from A to $\mathrm{B}$ independent of the entry station. Because $N_{A}+N_{A B} \gg N_{j_{A}}, P(A B) \cong P\left(A B \mid j_{A}\right)$, which precludes the need to calculate $P\left(A B \mid j_{A}\right)$ for every entry station. Similarly, the probability of not transferring from line A to B is

$$
1-P\left(A B \mid j_{A}\right)=\frac{N_{A}-N_{j_{A}}}{N_{A}+N_{A B}-N_{j_{A}}} \cong \frac{N_{A}}{N_{A}+N_{A B}}=1-P(A B) .
$$

The probability of exiting at the $i^{\text {th }}$ station on line B having entered at the $j^{\text {th }}$ station on line A and transferred via the $t^{\text {th }}$ station on line $\mathrm{B}$ is

$$
P\left(i_{B} \mid j_{A}\right)=\left(\frac{N_{i_{B}}}{N_{B}-N_{t_{B}}}\right) P\left(A B \mid j_{A}\right) \cong\left(\frac{N_{i_{B}}}{N_{B}-N_{t_{B}}}\right) P(A B)
$$

where $N_{t_{B}}$ is the number of people exiting at the transfer station(s).

\section{B.3 A Three-Line Subway (Multiple Transfer Stations)}

For a subway comprising three lines, A, B, and C, that have one (or more) transfer station(s) from $A$ to $B$ and from $B$ to $C$, the expression for a no-transfer trip is the same as for a 2-line subway above; the probability of exiting at the $i^{\text {th }}$ station on line $\mathrm{B}$ having entered at the $j^{\text {th }}$ station on line A and transferred to line $\mathrm{B}$ is

$$
\begin{gathered}
P\left(i_{B} \mid j_{A}\right)=\left(\frac{N_{i_{B}}}{N_{B}-N_{t_{B}}}\right) P\left(A B \mid j_{A}\right)\left(1-P\left(B C \mid t_{B}\right)\right) \\
\cong\left(\frac{N_{i_{B}}}{N_{B}-N_{t_{B}}}\right) P(A B)(1-P(B C)) .
\end{gathered}
$$

This is the product of the probability of exiting at the $i^{\text {th }}$ station on line B having originated at the A-to-B transfer station, the probability of transferring from line A to B, and the probability of not transferring from line B to C. Similarly, the probability of exiting at the $i^{\text {th }}$ station on line $\mathrm{C}$ having entered at the $j^{\text {th }}$ station on line A (requiring two transfers) is

$$
\begin{aligned}
P\left(i_{C} \mid j_{A}\right) & =\left(\frac{N_{i_{C}}}{N_{C}-N_{t_{C}}}\right) P\left(A B \mid j_{A}\right) P\left(B C \mid t_{B}\right) \\
& \cong\left(\frac{N_{i_{C}}}{N_{C}-N_{t_{C}}}\right) P(A B) P(B C) .
\end{aligned}
$$

Note that $P(A B) P(B C)=P(A C)$, the probability of transferring from A to $\mathrm{C}$. 


\section{B.4 A Four-Line Subway (Multiple Transfer Stations)}

For a subway comprising four lines, A, B, C, and D, that allow a transfer from A to B, A to C, B to $\mathrm{D}$, and $\mathrm{C}$ to $\mathrm{D}$, but not $\mathrm{A}$ to $\mathrm{D}$. (The MBTA subway is an example: the green and orange lines both intersect the blue and red lines, but the blue line and red line to not intersect):

No transfer:

$$
\begin{aligned}
P\left(i_{A} \mid j_{A}\right) & =\left(\frac{N_{i_{A}}}{N_{A}-N_{j_{A}}}\right)\left(1-\left(P\left(A B \mid j_{A}\right)+P\left(A C \mid j_{A}\right)\right)\right) \\
\cong & \left(\frac{N_{i_{A}}}{N_{A}-N_{j_{A}}}\right)(1-(P(A B)+P(A C))) .
\end{aligned}
$$

Single transfer:

$$
\begin{gathered}
P\left(i_{B} \mid j_{A}\right)=\left(\frac{N_{i_{B}}}{N_{B}-N_{t_{B}}}\right) P\left(A B \mid j_{A}\right)\left(1-P\left(B D \mid t_{B}\right)\right) \\
\cong\left(\frac{N_{i_{B}}}{N_{B}-N_{t_{B}}}\right) P(A B)(1-P(B D))
\end{gathered}
$$

Two transfers:

$$
\begin{gathered}
P\left(i_{D} \mid j_{A}\right)=\left(\frac{N_{i_{D}}}{N_{D}-N_{t_{D}}}\right)\left(P\left(A B \mid j_{A}\right) P\left(B D \mid t_{B}\right)+P\left(A C \mid j_{A}\right) P\left(C D \mid t_{C}\right)\right) \\
\cong\left(\frac{N_{i_{D}}}{N_{D}-N_{t_{D}}}\right)(P(A B) P(B D)+P(A C) P(C D)) \\
=\left(\frac{N_{i_{D}}}{N_{D}-N_{t_{D}}}\right) P(A D) .
\end{gathered}
$$

where

$$
P(A D)=P(A B) P(B D)+P(A C) P(C D)
$$

is the probability of a two-line transfer, $N_{t_{D}}$ is the combined number of people that exit at the Bto-D and C-to-D transfer stations, and

$$
\begin{aligned}
& P(A B)=\frac{N_{A B}}{N_{A}+N_{A B}+N_{A C}} ; P(A C)=\frac{N_{A C}}{N_{A}+N_{A B}+N_{A C}} ; \\
& P(B D)=\frac{N_{B C}}{N_{B}+N_{B C}+N_{C D}} ; P(C D)=\frac{N_{C D}}{N_{B}+N_{B C}+N_{C D}} .
\end{aligned}
$$


This page intentionally left blank. 


\section{Appendix C: Probit Analysis and Dose-Response Models}

The basis of probit analysis (Bliss 1934) is that the logarithm of the effective dosage ${ }^{1}$ required to cause a fraction $F$ of a population to exhibit a given health endpoint (e.g., incapacitation or mortality) may be represented by a cumulative normal distribution

$$
F(Y)=\frac{1}{\sqrt{2 \pi}} \int_{-\infty}^{Y} e^{-z^{2} / 2} d z
$$

This may be expressed in terms of the error function $\operatorname{erf}(x)$

$$
F(Y)=\frac{1}{2}\left(1+\operatorname{erf}\left[\frac{Y}{\sqrt{2}}\right]\right)
$$

$Y$ is a standard normal deviate or normit $^{2}$

$$
Y=\frac{\log _{10}(E C T)-\log _{10}\left(E C T_{50}\right)}{\sigma}=m \log _{10}\left(\frac{E C T}{E C T_{50}}\right)
$$

where $E C T$ is the effective dosage, which is the product of the concentration $C$ and the exposure time $T$. ECT 50 and $\sigma^{2}$ are, respectively, the median and variance of the distribution of $\log _{10}(E C T)$, and $m=1 / \sigma$ is the probit slope; the narrower the distribution of effective dosage, the larger the probit slope.

The probit slope and $E C T_{50}$ are typically determined by fitting toxicology data to the doseresponse model

$$
Y=m \log _{10}(E C T)+b
$$

to find $m$ and $b$. Noting $b=-m \log _{10}\left(E C T_{50}\right)$, then $E C T_{50}=10^{-b / m}$. Having values for $m$ and $E C T_{50}$, the effective dosage required to cause the fraction $F$ of a population to exhibit the specified biological response is

$$
E C T_{F}=E C T_{50} 10^{\mathrm{Y}(\mathrm{F}) / m}
$$

where $Y(F)$ is the normit function, which is the inverse of the cumulative normal distribution function

$$
Y(F)=\sqrt{2} \operatorname{erf}^{-1}(2 F-1)
$$

and $\operatorname{erf}^{-1}$ is the inverse error function.

1 The effective dosage is the threshold dosage that is just sufficient to cause the specified biological response in each individual.

2 For historical reasons (Bliss 1934), $Y+5$ is a probit (probability unit). 
For example, suppose a biological agent has a probit slope of 0.7 and $L C T_{50}=8000$ cfu (colonyforming units). Assuming $10^{9} \mathrm{cfu} \mathrm{mg}^{-1}$ and a breathing rate of $15 \mathrm{~L} \mathrm{~min}^{-1}$, this corresponds to $L C T_{50}=5.33 \times 10^{-4} \mathrm{mg} \mathrm{min}^{-3}$ or $3.20 \times 10^{-5} \mathrm{~g} \mathrm{~s} \mathrm{~m}^{-3}$. For $90 \%$ mortality, $Y(0.9)=1.28155$ and $L C T_{90}=3.61 \times 10^{-1} \mathrm{mg} \mathrm{min}^{-3}(5.4$ million cfu). For $10 \%$ mortality, $Y(0.1)=-1.28155$ and $L C T_{10}=7.87 \times 10^{-6} \mathrm{mg} \mathrm{min} \mathrm{m}^{-3}(118 \mathrm{cfu})$.

\section{C.1 Toxic Load}

The foregoing discussion is based on the dose-response relationship $C T=$ constant, known as Haber's Law. However, it has been observed that for some substances a short exposure to a high concentration can be more effective (i.e., worse) than a long exposure to a low concentration, even though the $C T$ values are the same. To accommodate this situation, a dose-response relationship of the form $C^{n} T=$ constant is used (ten Berge, Zwart, and Appelman 1986), where $n$ is referred to as the toxic load exponent. In this case, the dose-response data are fitted to the following form:

$$
Y=m_{c} \log _{10} C+m_{t} \log _{10} T+b \text {. }
$$

The toxic load exponent is defined by

$$
n=\frac{m_{c}}{m_{t}}
$$

Substituting and rearranging gives

$$
Y=\frac{m_{c}}{n} \log _{10}\left(E C^{n} T\right)+b
$$

Because $Y(F=0.5)=0$,

$$
b=-\frac{m_{c}}{n} \log _{10}\left(E C^{n} T_{50}\right)
$$

Then

$$
Y=\frac{m_{c}}{n} \log _{10}\left(\frac{E C^{n} T}{E C^{n} T_{50}}\right)
$$

Or

$$
E C^{n} T_{\mathrm{F}}=E C^{n} T_{50} 10^{n Y_{F} / m_{c}}
$$

where $m_{\mathrm{c}}$ is the probit slope. The value of $E C^{n} T_{50}$ may be calculated from the value for $E C T_{50}$ using 


$$
E C^{n} T_{50}=\left(\frac{E C T_{50}}{T}\right)^{n} T
$$

where $T$ is the exposure time used to determine the $E C T_{50}$ value.

For example, suppose a chemical agent has $m=12, n=1.5$, and $L C T_{50}=4200 \mathrm{mg} \mathrm{s} \mathrm{m}^{-3}$ based on a 2-minute exposure time. Then $L C^{n} T_{50}=(4200 / 120)^{1.5} \times 120=24848$. The following table lists a range of agent concentrations and exposure times that yield the same $C T$ value but different $C^{n} T$ values and correspondingly different mortality results.

Table 1. Toxic Load Example for $\mathrm{n}=1.5$.

\begin{tabular}{ccccc}
\hline $\begin{array}{c}\text { Concentration } \\
\left(\mathrm{mg} \mathrm{m}^{-3}\right)\end{array}$ & $\begin{array}{c}\text { Exposure Time } \\
(\mathrm{s})\end{array}$ & $\begin{array}{c}\mathrm{CT} \\
\left(\mathrm{mg} \mathrm{s} \mathrm{m}^{-3}\right)\end{array}$ & $\mathbf{C}^{\mathbf{n}} \mathbf{T}$ & \% Fatal \\
\hline 70 & 60 & 4200 & 35140 & 89 \\
35 & 120 & 4200 & 24848 & 50 \\
14 & 300 & 4200 & 15715 & 6 \\
7 & 600 & 4200 & 11112 & $<1$ \\
\hline
\end{tabular}


This page intentionally left blank. 


\section{Appendix D: Determination of Resuspension Rates}

The flux of resuspended material away from a surface onto which it has previously deposited is described by the resuspension rate:

$$
r=\frac{F_{r}}{S}
$$

where $F_{r}$ is the resuspension mass flux $\left(\mathrm{g} \mathrm{m}^{-2} \mathrm{~s}^{-1}\right)$ and $S$ is the surface mass loading $\left(\mathrm{g} \mathrm{m}^{-2}\right)$, such that $r$ has units of $\mathrm{s}^{-1}$. The resuspension rate subsumes the turbulent transport and the particlesurface interaction processes into a single constant of proportionality, which then depends on the material being resuspended, the surface from which it is resuspending, and the conditions under which the resuspension takes place. For this reason, it is important to choose resuspension rates that are appropriate for the material, surface, and conditions of interest.

\section{D.1 Resuspension from Surfaces}

For resuspension from the interior surfaces of the train cars, especially the floors and seats, we use resuspension rates determined by Freihaut, Bahnfleth, and $\mathrm{Hu}$ (2008). They placed samples of linoleum flooring and commercial-grade low-pile carpet with a known initial mass loading in a chamber equipped with a mechanical vibrator and small air jets to allow the effects of footsteps to be simulated in a controlled manner. They investigated different particulate materials (quartz dust, dust mite, cat dander, and dog dander), particle sizes, airflow rates (to simulate air motion caused by ventilation and people walking), humidity levels, and initial mass loadings. Their results showed a large initial resuspension rate followed by a rapid decay, suggesting that as more people walk through an area, less material is resuspended. This rapid decay in the resuspension rate is consistent with results for bare soil, concrete, and grass surfaces in a wind tunnel, presented by Loosmore (2003). Freihaut et al. (2008) also demonstrated a strong dependence on airflow, with resuspension rates for airflow of $0.3 \mathrm{~m} \mathrm{~s}^{-1}$ (typical of ventilationinduced flow) about two orders of magnitude less than for $1.5 \mathrm{~m} \mathrm{~s}^{-1}$ (typical walking speed). This is also consistent with Loosmore, who found a strong dependence of resuspension rate on friction velocity (surface stress), which increases with airflow. In addition, Freihaut et al. found that Bacillus thuringiensis $(B t)$ spores were much more easily resuspended than either quartz dust or mite particles. Accordingly, we use their 2-minute average resuspension rates for mite particles, adjusted for $B t$, with a $1.5 \mathrm{~m} \mathrm{~s}^{-1}$ airflow to describe the resuspension from floors by patrons' foot traffic; we use the peak rates (an order of magnitude greater than the average rates) to describe resuspension from seats when passengers sit down. These resuspension rates are comparable to those for vinyl flooring and carpet obtained by Qian and Ferro (2008) for human subjects walking and sitting in a controlled chamber. In contrast to Loosmore, who found only a weak dependence on particle size, the studies of Freihaut et al. and Qian and Ferro reveal a strong dependence of resuspension rate on particle size, which is consistent with particle resuspension arising from human activities in a residence reported by Thatcher and Layton (1995). 


\section{D.2 Resuspension from People}

There are few studies of particle resuspension from people, and fewer still that provide estimates of resuspension rates. Hession et al. (2006) measured resuspension rates for $3-\mu \mathrm{m}$ and $10-\mu \mathrm{m}$ silica particles from the bare forearms of volunteers seated working at a desk or walking "vigorously." Whereas particles were resuspended from the seated volunteer at a steady rate over a 6-hour period, for the vigorously walking volunteer, $88 \%$ of the initial mass was lost after only 30 minutes, with very little additional resuspension measured thereafter. This result suggests that higher airflow, producing higher surface stress, causes more particles to be resuspended, which is consistent with the findings of Freihaut et al. and Loosmore. Hession et al. also found very little effect of particle size on the resuspension rate, which is also consistent with Loosmore. Recently McDonagh and Byrne (2014a, 2014b) determined the fraction of silica particles resuspended from fabric samples attached to a volunteer in a chamber after 20 minutes of slow walking or 10 minutes of Irish dancing (to simulate running) for 3-, 5-, and 10- $\mu \mathrm{m}$ particles. Consistent with Loosmore and Hession et al., their results exhibited only a slight dependence on particle size. McDonagh and Byrne also measured a moderately higher resuspended fraction for fleece, with polyester, denim, and cotton all producing comparable resuspended fractions. Accordingly, we neglect particle size and fabric type as factors in the resuspension rate from people's clothing.

Average resuspension rates may be determined from McDonagh and Byrne's resuspended fractions by dividing by the period of activity. For walking, this yields a rate about twice that of Hession et al.'s average rate for vigorous walking. For dancing (simulating running), McDonagh and Byrne's average resuspension rate is comparable to the rate calculated from Hession et al.'s results for the first 30 minutes of vigorous walking. Consequently, we use Hession et al.'s average seated resuspension rate to describe resuspension from stationary subway patrons (either standing or seated); for patrons boarding or alighting from a train or exiting the subway, we use the average rate derived from McDonagh and Byrne's resuspended fraction by walking. These values are summarized in Table 2 .

Table 2. Summary of Resuspension Rates

\begin{tabular}{lcc}
\hline \multicolumn{1}{c}{ Condition } & Particle Size, $\mu \mathrm{m}$ & $\begin{array}{c}\text { Resuspension Rate, } \\
\text { min }^{-1}\end{array}$ \\
\hline From surfaces, by ventilation & 3 & $1.3 \times 10^{-6}$ \\
From floors, by walking & 3 & $1.3 \times 10^{-4}$ \\
From seats, by sitting & 3 & $1.3 \times 10^{-3}$ \\
From persons standing/sitting & All sizes & $1.5 \times 10^{-3}$ \\
From persons walking & All sizes & $1.3 \times 10^{-2}$ \\
\hline
\end{tabular}





\section{Argonne}

Decision and Information Sciences Division

Argonne National Laboratory

9700 South Cass Avenue, Bldg. 221

Argonne, IL 60439-4844

www.anl.gov

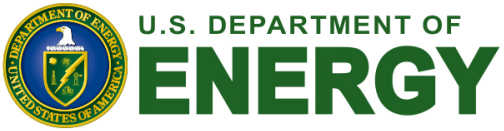

Argonne National Laboratory is a U.S. Department of Energy

laboratory managed by UChicago Argonne, LLC 